Article

\title{
Hybrid Control Scheme for Projective Lag Synchronization of Riemann-Liouville Sense Fractional Order Memristive BAM Neural Networks with Mixed Delays
}

\author{
Grienggrai Rajchakit ${ }^{1}$, Anbalagan Pratap ${ }^{2}$, Ramachandran Raja ${ }^{3}$, Jinde Cao ${ }^{4, *(\mathbb{D})}$ \\ Jehad Alzabut ${ }^{5}(\mathbb{C})$ and Chuangxia Huang ${ }^{6}(\mathbb{C})$ \\ 1 Department of Mathematics, Maejo University, Chiangmai 50290, Thailand \\ 2 Vel Tech High Tech Dr Rangarajan Dr Sakunthala Engineering College, Avadi 600 062, India \\ 3 Ramanujan Centre for Higher Mathematics, Alagappa University, Karaikudi 630 004, India \\ 4 School of Mathematics, Southeast University, Nanjing 211189, China \\ 5 Department of Mathematics and General Sciences, Prince Sultan University, 11586 Riyadh, Saudi Arabia \\ 6 Hunan Provincial Key Laboratory of Mathematical Modeling and Analysis in Engineering, Department of \\ Applied Mathematics, Changsha University of Science and Technology, Changsha 410114, China \\ * Correspondence: jdcao@seu.edu.cn
}

Received: 12 July 2019; Accepted: 14 August 2019; Published: 19 August 2019

check for updates

\begin{abstract}
This sequel is concerned with the analysis of projective lag synchronization of Riemann-Liouville sense fractional order memristive BAM neural networks (FOMBNNs) with mixed time delays via hybrid controller. Firstly, a new type of hybrid control scheme, which is the combination of open loop control and adaptive state feedback control is designed to guarantee the global projective lag synchronization of the addressed FOMBNNs model. Secondly, by using a Lyapunov-Krasovskii functional and Barbalet's lemma, a new brand of sufficient criterion is proposed to ensure the projective lag synchronization of the FOMBNNs model considered. Moreover, as special cases by using a hybrid control scheme, some sufficient conditions are derived to ensure the global projective synchronization, global complete synchronization and global anti-synchronization for the FOMBNNs model considered. Finally, numerical simulations are provided to check the accuracy and validity of our obtained synchronization results.
\end{abstract}

Keywords: memristive BAM neural networks; hybrid control; mixed delays; synchronization

\section{Introduction}

Differential equation and dynamic system modeling have become important research topics in natural science and engineering technology [1-12]. In 1695, the foundation of non-integer order calculus, which is a generalization integer order differential and integrals, was first of all discussed through Guillaume de Leibnitz and Gottfried Wilhelm Leibnitz, and its development were very gradual for long period [13-16]. However, during the last two decades, the study of fractional differential equations has been widely applicable to many real world problems. In fact, they have already been successfully applied in many fields of engineering including but not restricted to market dynamics [17], neural networks [18] and polarization [19]. In reality, many real-world objects need to 
be described with the aid of fractional order models due to the fact that dynamics of fractional-order models are more correct than integer-order models. From the application point of view, for the electronic implementation of bidirectional associative memory (BAM) neural network model, many of the researchers attempted to update the normal capacitor by fractional capacitor; then, it creates the fractional order BAM neural network (FOBNNs) models [20-24]. In addition to that, time delays can impose complexity and restrictions in neural networks and the existence may lead to instability, chaos, and oscillation [25-34]. At present, a huge amount of research works on the dynamic FOBNNs with time delays have been discussed. For example, in [20], the authors pointed out the global asymptotical stability criteria of fuzzy FOBNNs with delays and impulsive effects by Riemann-Liouville derivative, employing contraction mapping principle and fuzzy theory. In [21], the authors illustrate the global asymptotic stability issues of FOBNNs with delays and impulses via fractional order Barbalat's lemma and Lyapunov-like function. Otherwise, during a particular period, the signal propagation is distributed because the variety of axon sizes and lengths are too large. In this manner, the attention of distributed delays is significant in fractional order neural networks (FONNs) dynamical systems, and there is a huge amount of research works on FONNs with distributed delay-see, for instance, [35,36]. In [20], the existence and Lyapunov-stability of impulsive hybrid FOBNNs with mixed delays were discussed by Zhang et al. and the sufficient conditions are derived to assure the stability condition of Hybrid FOBNNs with mixed time-delays via contraction mapping principle, integer order Lyapunov functional and periodically intermittent control.

In pattern recognition, optimization, and associative memory, memristors have the most crucial probable applications, which are introduced by Leon Chua in 1971 [37]. Chua's paper in IEEE Transactions on Circuit theory in 1971 is considered to be the pioneering work in the corresponding subject of studies, after almost 40 years, for memristors to change from a definitely theoretic idea into workable usage. The Hewlett-Packard laboratory established the practically working memristor in 2008 [38]. In [39], Kim et al. successfully proposed memristor bridge synapse architecture and resolved the difficulty of the problem of nonvolatile synaptic weight garage and put in force recently proposed hardware learning techniques. After this, the memristor has discovered numerous applications in various interdisciplinary areas. The resistor is used to simulate the biological synapses of neural networks circuit implementation, and these biological synapses have the same characteristics as those of a memristor. Then, by replacing the resistor with a memristor in fractional order neural networks circuit implementation, the fractional order memristive neural networks (FOMNNs) can be formulated. Still now, via different approaches, many novel results on the dynamical analysis of FOMNNs time delays have been reported in [40-43].

The basic idea of chaos synchronization with different initial conditions was initially introduced by Pecora and Carroll in [44], and it has attracted great attention due to their successful applications such as artificial intelligence, image processing automatic control, associative memories, and secure communication. A few coupled structures can synchronize by themselves. However, others can't attain synchronization by themselves. On this circumstance, we have applied some suitable controllers to practical fractional order neural networks system to make certain the corresponding asymptotic behavior and enhance system performance. During the recent years, various kinds of control techniques have been developed by many researchers, for instance, [34,45-50]. In [34], by using the adaptive control and linear delayed feedback control technique, the author discussed the synchronization of delayed FOMNNs with different orders. In [48], Wu et al. presented some new conditions on the projective synchronization in finite time of nonidentical neural networks with fractional order derivative by using sliding mode control theory. In [49], the sufficient conditions are derived to ensure the finite-time Mittag-Leffler synchronization of delayed FOMNNs with different 
orders by applying the Lyapunov-stability theory and a state feedback control. In Ref. [50], the authors addressed the lag synchronization for FOMNNs on the basis of period intermittent control, properties of Mittag-Leffler function and fractional inequality technique. Nevertheless, the synchronization of FOMBNNs via a hybrid control approach has not yet been investigated. Motivated by the above arguments, the main novelty of this research work is outlined in detail as follows:

1. Based on the theory of differential inclusions and set valued map analysis, the drive-response synchronization error system is formulated.

2. A novel hybrid controller, which is the combination of open loop control and adaptive state feedback control are designed to ensure the projective lag synchronization criteria for FOMBNNs with mixed time delays.

3. Based on the designed hybrid controller and Barbalats lemma, the projective lag synchronization criteria for the drive-response models of the considered FOMBNNs are studied demonstrably.

4. As a special case of complete synchronization, anti-synchronization and projective synchronization of FMNNs is also investigated. Hence, Corollary 3 is new and these results has not been seen in any of the literature.

5. In contrast to the existing results in the literature, the hybrid control BAM type neural networks and mixed time delays have not taken into consideration; however, our proposed results make it up.

\section{Preliminaries and Problem Statement}

Notations. In this work, $N$ signifies the space of natural numbers from 1 to $n, R^{n}$ stands for the space of $n$-D Euclidean space, respectively. $R^{m \times n}$ stands for the set of all $m \times n$ real matrices. For $z(t)=\left(z_{1}(t), \ldots, z_{n}(t)\right)^{T} \in R^{n}$, we denote

$$
\|z(t)\|=\sum_{i=1}^{n}\left|z_{i}(t)\right|
$$

The sign function $\operatorname{sign}(z)$ is described by

$$
\operatorname{sign}(z)=\left\{\begin{array}{r}
1, z>0 \\
0, z=0 \\
-1, \quad z<0
\end{array}\right.
$$

Let $C\left([-\omega, 0], R^{n}\right)$ describe the family of Banach space, which consists of 1-order continuous function maps from $[-\omega, 0]$ into $R^{n}$. $D^{q}$ denotes the Riemann-Liouville derivative operator with $0<q<1 . \mathcal{K}[L]$ signifies the closure of the convex hull of set $L$.

In this section, we will first recall some basic definitions and important properties in fractional order calculus.

Definition 1. The Riemann-Liouville fractional integral of $z(t)$ is described as [15]:

$$
D^{-q} z(t)=\frac{1}{\Gamma(q)} \int_{0}^{t}(t-\gamma)^{q-1} z(\gamma) \mathrm{d} \gamma,
$$

where $q \in R^{+}$. 
Definition 2. The Caputo-type fractional derivative of $z(t)$ is described as [15]:

$$
D^{q} z(t)= \begin{cases}D^{-(n-q)}\left(\frac{d^{n}}{d t^{n}} z(t)\right), & \text { if } q \in(n-1, n), \\ \left(\frac{d^{n}}{d t^{n}} z(t)\right), & \text { if } q=n,\end{cases}
$$

where $q \in R^{+}, n \in Z^{+}$.

Definition 3. The Riemann-Liouville fractional derivative of $z(t)$ is described as [15]:

$$
\begin{aligned}
D^{q} z(t) & =\frac{d^{n}}{d t^{n}}\left[D^{(n-q)} z(t)\right] \\
& =\frac{1}{\Gamma(n-\alpha)} \frac{d^{n}}{d t^{n}} \int_{0}^{t}(t-\gamma)^{n-1-\alpha} z(\gamma) d \gamma,
\end{aligned}
$$

where $0 \leq n-1 \leq q<n, n \in Z^{+}$.

Lemma 1. Let $p>0, q>0$, and $x(t), z(t) \in C^{1}[0, b]$. The following properties hold [15]:

(1) $D^{p} D^{-q} x(t)=D^{p-q} x(t)$;

(2) $D^{p} D^{-p} x(t)=x(t)$;

(3) $D^{p}(x(t) \pm z(t))=D^{p} x(t) \pm D^{p} z(t)$.

Lemma 2. Function $\xi(t)$ is defined on the interval $[0,+\infty)$, if function $\xi(t)$ is uniformly continuous and $\int_{0}^{+\infty} \xi(\tau) d \tau$ exists and is bounded, then $\lim _{t \rightarrow+\infty} \xi(t)=0$ [51].

Motivated by the descriptions in the section above, in this work, we will firstly establish the model of FOMBNNs that can be represented as below:

$$
\begin{aligned}
D^{q} y_{i}(t)=- & a_{i} y_{i}(t)+\sum_{j=1}^{m}\left[b_{j i}\left(y_{i}(t)\right)\right] f_{j}\left(z_{j}(t)\right)+\sum_{j=1}^{m}\left[c_{j i}\left(y_{i}(t)\right)\right] f_{j}\left(z_{j}(t-\rho(t))\right) \\
& +\sum_{j=1}^{m}\left[d_{j i}\left(y_{i}(t)\right)\right] \int_{t-r(t)}^{t} f_{j}\left(z_{j}(s)\right) d s+J_{i}, t \geq 0, \\
D^{q} z_{j}(t)=- & u_{j} z_{j}(t)+\sum_{i=1}^{n}\left[v_{i j}\left(z_{j}(t)\right)\right] g_{i}\left(y_{i}(t)\right)+\sum_{i=1}^{n}\left[w_{i j}\left(z_{j}(t)\right)\right] g_{i}\left(y_{i}(t-\rho(t))\right) \\
& +\sum_{i=1}^{n}\left[x_{i j}\left(z_{j}(t)\right)\right] \int_{t-r(t)}^{t} g_{i}\left(y_{i}(s)\right) d s+H_{j}, t \geq 0,
\end{aligned}
$$

where $i \in\{1,2, \ldots, n\}$ and $j \in\{1,2, \ldots, m\}$. Let $y(t)=\left(y_{1}(t), y_{2}(t), \ldots, y_{n}(t)\right)^{T} \in R^{n}$ and $z(t)=$ $\left(z_{1}(t), z_{2}(t), \ldots, z_{m}(t)\right)^{T} \in R^{m}$ stands for the neural state vector at time t. $a_{i}>0$ and $u_{j}>0$ signifies the weight of self feedback connection. $f(z(t))=\left(\left(f_{1}\left(z_{1}(t)\right), \ldots,\left(f_{m}\left(z_{m}(t)\right)\right)^{T} \in R^{m}\right.\right.$ and $g(y(t))=\left(\left(g_{1}\left(y_{1}(t)\right), \ldots,\left(g_{n}\left(y_{n}(t)\right)\right)^{T} \in R^{n}\right.\right.$ represents the activation function of the neurons. $J_{i}$ and $H_{j}$ denotes external inputs outside the system and $\rho(t)>0$ corresponds to the transmission discrete delays and satisfies $0<\rho(t) \leq \rho, \dot{\rho}(t) \leq \sigma<1, \rho$ is constant, while $r(t)$ signifies the distributed time-varying delays, which is continuous function and satisfies $0<r(t) \leq r, r$ is positive constant. The memristor connection weights are defined by 


$$
\begin{aligned}
& b_{j i}\left(y_{i}(t)\right)=\left\{\begin{array}{l}
b_{j i,}^{\star},\left|y_{i}(t)\right| \leq T_{i} \\
b_{j i}^{\star \star},\left|y_{i}(t)\right|>T_{i},
\end{array} \quad c_{j i}\left(y_{i}(t)\right)=\left\{\begin{array}{l}
c_{j i^{\prime}}^{\star},\left|y_{i}(t)\right| \leq T_{i} \\
c_{j i}^{\star \star},\left|y_{i}(t)\right|>T_{i},
\end{array}\right.\right. \\
& d_{j i}\left(y_{i}(t)\right)=\left\{\begin{array}{l}
d_{j i^{\prime}}^{\star},\left|y_{i}(t)\right| \leq T_{i} \\
d_{j i}^{\star \star},\left|y_{i}(t)\right|>T_{i},
\end{array} \quad v_{i j}\left(z_{j}(t)\right)=\left\{\begin{array}{l}
v_{i j^{\prime}}^{\star},\left|z_{j}(t)\right| \leq \tilde{T}_{j} \\
v_{i j}^{\star \star},\left|z_{j}(t)\right|>\tilde{T}_{j},
\end{array}\right.\right. \\
& w_{i j}\left(z_{j}(t)\right)=\left\{\begin{array}{l}
w_{i j^{\prime}}^{\star}\left|z_{j}(t)\right| \leq \tilde{T}_{j} \\
w_{i j^{\star \star},}^{\star \star},\left|z_{j}(t)\right|>\tilde{T}_{j},
\end{array} \quad x_{i j}\left(z_{j}(t)\right)=\left\{\begin{array}{l}
x_{i j^{\prime}}^{\star},\left|z_{j}(t)\right| \leq \tilde{T}_{j} \\
x_{i j^{\star \star},},\left|z_{j}(t)\right|>\tilde{T}_{j},
\end{array}\right.\right.
\end{aligned}
$$

in which switching jumps $T_{i}, \tilde{T}_{j}>0, b_{j i}^{\star}, c_{j i}^{\star}, d_{j i}^{\star}, v_{i j}^{\star}, w_{i j}^{\star}, x_{i j}^{\star}, b_{j i}^{\star \star}, c_{j i}^{\star \star}, d_{j i}^{\star \star}, v_{i j}^{\star \star}, w_{i j}^{\star \star}$ and $x_{i j}^{\star \star}$ are known positive constants.

According to set-valued map theory and differential inclusion analysis [52,53], FOMBNNs (1) can be written as follows:

$$
\begin{aligned}
D^{q} y_{i}(t) \in \quad-a_{i} y_{i}(t)+\sum_{j=1}^{m}\left[\mathcal{K}\left(b_{j i}\left(y_{i}(t)\right)\right)\right] f_{j}\left(z_{j}(t)\right)+\sum_{j=1}^{m}\left[\mathcal{K}\left(c_{j i}\left(y_{i}(t)\right)\right)\right] f_{j}\left(z_{j}(t-\rho(t))\right) \\
\quad+\sum_{j=1}^{m}\left[\mathcal{K}\left(d_{j i}\left(y_{i}(t)\right)\right)\right] \int_{t-r(t)}^{t} f_{j}\left(z_{j}(s)\right) d s+J_{i}, t \geq 0 \\
D^{q} z_{j}(t) \in \quad-u_{j} z_{j}(t)+\sum_{i=1}^{n}\left[\mathcal{K}\left(v_{i j}\left(z_{j}(t)\right)\right)\right] g_{i}\left(y_{i}(t)\right)+\sum_{i=1}^{n}\left[\mathcal{K}\left(w_{i j}\left(z_{j}(t)\right)\right)\right] g_{i}\left(y_{i}(t-\rho(t))\right) \\
+\sum_{i=1}^{n}\left[\mathcal{K}\left(x_{i j}\left(z_{j}(t)\right)\right)\right] \int_{t-r(t)}^{t} g_{i}\left(y_{i}(s)\right) d s+H_{j}, t \geq 0 .
\end{aligned}
$$

We define

$$
\begin{aligned}
& \mathcal{K}\left[b_{j i}\left(y_{i}(t)\right)\right]=\left\{\begin{array}{ll}
b_{j i}^{\star}, & \left|y_{i}(t)\right| \leq T_{i} \\
{\left[\underline{b}_{j i}, \bar{b}_{j i}\right],} & \left|y_{i}(t)\right|=T_{i} \\
b_{j i}^{\star \star}, & \left|y_{i}(t)\right|>T_{i},
\end{array} \quad \mathcal{K}\left[c_{j i}\left(y_{i}(t)\right)\right]= \begin{cases}c_{j i}^{\star}, & \left|y_{i}(t)\right| \leq T_{i} \\
{\left[\underline{c}_{j i}, \bar{c}_{j i}\right],} & \left|y_{i}(t)\right|=T_{i} \\
c_{j i}^{\star \star}, & \left|y_{i}(t)\right|>T_{i},\end{cases} \right. \\
& \mathcal{K}\left[d_{j i}\left(y_{i}(t)\right)\right]=\left\{\begin{array}{ll}
d_{j i}^{\star}, & \left|y_{i}(t)\right| \leq T_{i} \\
{\left[\underline{d}_{j i}, \bar{d}_{j i}\right],} & \left|y_{i}(t)\right|=T_{i} \\
d_{j i}^{\star \star}, & \left|y_{i}(t)\right|>T_{i},
\end{array} \quad \mathcal{K}\left[v_{i j}\left(z_{j}(t)\right)\right]= \begin{cases}v_{i j}^{\star}, & \left|z_{j}(t)\right| \leq \tilde{T}_{j} \\
{\left[\underline{v}_{i j}, \bar{v}_{i j}\right],} & \left|z_{j}(t)\right|=\tilde{T}_{j} \\
v_{i j}^{\star \star}, & \left|z_{j}(t)\right|>\tilde{T}_{j,}\end{cases} \right. \\
& \mathcal{K}\left[w_{i j}\left(z_{j}(t)\right)\right]=\left\{\begin{array}{ll}
w_{i j \prime}^{\star}, & \left|z_{j}(t)\right| \leq \tilde{T}_{j} \\
{\left[w_{i j}, \bar{w}_{i j}\right],} & \left|z_{j}(t)\right|=\tilde{T}_{j} \\
w_{i j}^{\star \star}, & \left|z_{j}(t)\right|>\tilde{T}_{j,}
\end{array} \quad \mathcal{K}\left[x_{i j}\left(z_{j}(t)\right)\right]=\left\{\begin{array}{l}
x_{i j^{\prime}}^{\star}, \quad\left|z_{j}(t)\right| \leq \tilde{T}_{j} \\
{\left[x_{i j}, \bar{x}_{i j}\right],\left|z_{j}(t)\right|=\tilde{T}_{j}} \\
x_{i j}^{\star \star}, \quad\left|z_{j}(t)\right|>\tilde{T}_{j} .
\end{array}\right.\right.
\end{aligned}
$$


In this work, we define $\underline{b}_{j i}=\min \left\{b_{j i}^{\star}, b_{j i}^{\star \star}\right\}, \bar{b}_{j i}=\max \left\{b_{j i}^{\star}, b_{j i}^{\star \star}\right\}, \underline{c}_{j i}=\min \left\{c_{j i}^{\star}, c_{j i}^{\star \star}\right\}, \bar{c}_{j i}=$ $\max \left\{c_{j i}^{\star}, c_{j i}^{\star \star}\right\}, \underline{d}_{j i}=\min \left\{d_{j i}^{\star}, d_{j i}^{\star \star}\right\}, \bar{d}_{j i}=\max \left\{d_{j i}^{\star}, d_{j i}^{\star \star}\right\}, \underline{v}_{i j}=\min \left\{v_{i j}^{\star}, v_{i j}^{\star \star}\right\}, \bar{v}_{i j}=\max \left\{v_{i j}^{\star}, v_{i j}^{\star \star}\right\}$, $\underline{w}_{i j}=\min \left\{w_{i j}^{\star}, w_{i j}^{\star \star}\right\}, \bar{w}_{i j}=\max \left\{w_{i j}^{\star}, w_{i j}^{\star \star}\right\}, \bar{x}_{i j}=\max \left\{x_{i j}^{\star}, x_{i j}^{\star \star}\right\}, \underline{x}_{i j}=\min \left\{x_{i j}^{\star}, x_{i j}^{\star \star}\right\}$.

Then, there exist $\hat{b}_{j i}(t) \in\left[\mathcal{K}\left(b_{j i}\left(y_{i}(t)\right)\right)\right], \hat{c}_{j i}(t) \in\left[\mathcal{K}\left(c_{j i}\left(y_{i}(t)\right)\right)\right], \hat{a}_{j i}(t) \in\left[\mathcal{K}\left(d_{j i}\left(y_{i}(t)\right)\right)\right], \hat{v}_{i j}(t) \in$ $\left[\mathcal{K}\left(v_{i j}\left(z_{j}(t)\right)\right)\right], \hat{w}_{i j}(t) \in\left[\mathcal{K}\left(w_{i j}\left(z_{j}(t)\right)\right)\right]$ and $\hat{x}_{i j}(t) \in\left[\mathcal{K}\left(x_{i j}\left(z_{j}(t)\right)\right)\right]$, the measurable function such that

$$
\begin{array}{r}
D^{q} y_{i}(t)=-a_{i} y_{i}(t)+\sum_{j=1}^{m} \hat{b}_{j i}(t) f_{j}\left(z_{j}(t)\right)+\sum_{j=1}^{m} \hat{c}_{j i}(t) f_{j}\left(z_{j}(t-\rho(t))\right)+\sum_{j=1}^{m} \hat{d}_{j i}(t) \int_{t-r(t)}^{t} f_{j}\left(z_{j}(s)\right) d s \\
+J_{i}, t \geq 0 \\
D^{q} z_{j}(t)=-u_{j} z_{j}(t)+\sum_{i=1}^{n} \hat{v}_{i j}(t) g_{i}\left(y_{i}(t)\right)+\sum_{i=1}^{n} \hat{w}_{i j}(t) g_{i}\left(y_{i}(t-\rho(t))\right)+\sum_{i=1}^{n} \hat{x}_{i j}(t) \int_{t-r(t)}^{t} g_{i}\left(y_{i}(s)\right) d s \\
+H_{j}, t \geq 0 .
\end{array}
$$

Consider FOMBNNs (1) as a drive system, the response system is given by

$$
\begin{aligned}
D^{q} \tilde{y}_{i}(t)=- & a_{i} \tilde{y}_{i}(t)+\sum_{j=1}^{m}\left[b_{j i}\left(\tilde{y}_{i}(t)\right)\right] f_{j}\left(\tilde{z}_{j}(t)\right)+\sum_{j=1}^{m}\left[c_{j i}\left(\tilde{y}_{i}(t)\right)\right] f_{j}\left(\tilde{z}_{j}(t-\rho(t))\right) \\
& +\sum_{j=1}^{m}\left[d_{j i}\left(\tilde{y}_{i}(t)\right)\right] \int_{t-r(t)}^{t} f_{j}\left(\tilde{z}_{j}(s)\right) d s+J_{i}+\zeta_{i}(t), t \geq 0 \\
D^{q} \tilde{z}_{j}(t)=- & u_{j} \tilde{z}_{j}(t)+\sum_{i=1}^{n}\left[v_{i j}\left(\tilde{z}_{j}(t)\right)\right] g_{i}\left(\tilde{y}_{i}(t)\right)+\sum_{i=1}^{n}\left[w_{i j}\left(\tilde{z}_{j}(t)\right)\right] g_{i}\left(\tilde{y}_{i}(t-\rho(t))\right) \\
& +\sum_{i=1}^{n}\left[x_{i j}\left(\tilde{z}_{j}(t)\right)\right] \int_{t-r(t)}^{t} g_{i}\left(\tilde{y}_{i}(s)\right) d s+H_{j}+\varsigma_{j}(t) t \geq 0 .
\end{aligned}
$$

for $i \in\{1,2, \ldots, n\}$ and $j \in\{1,2, \ldots, m\}$, where $\zeta_{i}(t)$ and $\varsigma_{j}(t)$ denotes the hybrid control. Let $\tilde{y}(t)=$ $\left(\tilde{y}_{1}(t), \tilde{y}_{2}(t), \ldots, \tilde{y}_{n}(t)\right)^{T} \in R^{n}$ and $\tilde{z}(t)=\left(\tilde{z}_{1}(t), \tilde{z}_{2}(t), \ldots, \tilde{z}_{m}(t)\right)^{T} \in R^{m}$ is the neuron state vector of the response system (4), the parameters can be defined as

$$
\begin{aligned}
& b_{j i}\left(\tilde{y}_{i}(t)\right)=\left\{\begin{array}{l}
b_{j i}^{\star},\left|\tilde{y}_{i}(t)\right| \leq T_{i} \\
b_{j i}^{\star \star},\left|\tilde{y}_{i}(t)\right|>T_{i},
\end{array} \quad c_{j i}\left(\tilde{y}_{i}(t)\right)=\left\{\begin{array}{l}
c_{j i}^{\star},\left|\tilde{y}_{i}(t)\right| \leq T_{i} \\
c_{j i}^{\star \star},\left|\tilde{y}_{i}(t)\right|>T_{i},
\end{array}\right.\right. \\
& d_{j i}\left(\tilde{y}_{i}(t)\right)=\left\{\begin{array}{ll}
d_{j i}^{\star}, & \left|\tilde{y}_{i}(t)\right| \leq T_{i} \\
d_{j i}^{\star \star}, & \left|\tilde{y}_{i}(t)\right|>T_{i},
\end{array} \quad v_{i j}\left(\tilde{z}_{j}(t)\right)= \begin{cases}v_{i j}^{\star}, & \left|\tilde{z}_{j}(t)\right| \leq \tilde{T}_{j} \\
v_{i j}^{\star \star}, & \left|\tilde{z}_{j}(t)\right|>\tilde{T}_{j},\end{cases} \right. \\
& w_{i j}\left(\tilde{z}_{j}(t)\right)= \begin{cases}w_{i j}^{\star},\left|\tilde{z}_{j}(t)\right| \leq \tilde{T}_{j} \\
w_{i j}^{\star \star}, & \left|\tilde{z}_{j}(t)\right|>\tilde{T}_{j,},\end{cases}
\end{aligned}
$$


By using differential inclusion of response system FOMBNNs (5), in the sense of Filippov, it follows that

$$
\begin{aligned}
D^{q} \tilde{y}_{i}(t) \in \quad-a_{i} \tilde{y}_{i}(t)+\sum_{j=1}^{m}\left[\mathcal{K}\left(b_{j i}\left(\tilde{y}_{i}(t)\right)\right)\right] f_{j}\left(\tilde{z}_{j}(t)\right)+\sum_{j=1}^{m}\left[\mathcal{K}\left(c_{j i}\left(\tilde{y}_{i}(t)\right)\right)\right] f_{j}\left(\tilde{z}_{j}(t-\rho(t))\right) \\
\quad+\sum_{j=1}^{m}\left[\mathcal{K}\left(d_{j i}\left(\tilde{y}_{i}(t)\right)\right)\right] \int_{t-r(t)}^{t} f_{j}\left(\tilde{z}_{j}(s)\right) d s+J_{i}+\zeta_{i}(t), t \geq 0 \\
D^{q} \tilde{z}_{j}(t) \in \quad-u_{j} \tilde{z}_{j}(t)+\sum_{i=1}^{n}\left[\mathcal{K}\left(v_{i j}\left(\tilde{z}_{j}(t)\right)\right)\right] g_{i}\left(\tilde{y}_{i}(t)\right)+\sum_{i=1}^{n}\left[\mathcal{K}\left(w_{i j}\left(\tilde{z}_{j}(t)\right)\right)\right] g_{i}\left(\tilde{y}_{i}(t-\rho(t))\right) \\
\quad+\sum_{i=1}^{n}\left[\mathcal{K}\left(x_{i j}\left(\tilde{z}_{j}(t)\right)\right)\right] \int_{t-r(t)}^{t} g_{i}\left(\tilde{y}_{i}(s)\right) d s+H_{j}+\varsigma_{j}(t), t \geq 0 .
\end{aligned}
$$

We define

$$
\begin{aligned}
& \mathcal{K}\left[b_{j i}\left(\tilde{y}_{i}(t)\right)\right]=\left\{\begin{array}{ll}
b_{j i}^{\star}, & \left|\tilde{y}_{i}(t)\right| \leq \tilde{T}_{j} \\
{\left[\underline{b}_{j i}, \bar{b}_{j i}\right],} & \left|\tilde{y}_{i}(t)\right|=\tilde{T}_{j} \\
b_{j i}^{\star \star}, & \left|\tilde{y}_{i}(t)\right|>\tilde{T}_{j,}
\end{array} \quad \mathcal{K}\left[c_{j i}\left(\tilde{y}_{i}(t)\right)\right]= \begin{cases}c_{j i}^{\star}, & \left|\tilde{y}_{i}(t)\right| \leq \tilde{T}_{j} \\
{\left[\underline{c}_{j i}, \bar{c}_{j i}\right],} & \left|\tilde{y}_{i}(t)\right|=\tilde{T}_{j} \\
c_{j i}^{\star \star}, & \left|\tilde{y}_{i}(t)\right|>\tilde{T}_{j,}\end{cases} \right. \\
& \mathcal{K}\left[d_{j i}\left(\tilde{y}_{i}(t)\right)\right]=\left\{\begin{array}{ll}
d_{j i}^{\star}, & \left|\tilde{y}_{i}(t)\right| \leq \tilde{T}_{j} \\
{\left[\underline{d}_{j i}, \bar{d}_{j i}\right],} & \left|\tilde{y}_{i}(t)\right|=\tilde{T}_{j} \\
d_{j i}^{\star \star}, & \left|\tilde{y}_{i}(t)\right|>\tilde{T}_{j,}
\end{array} \quad \mathcal{K}\left[v_{i j}\left(\tilde{z}_{j}(t)\right)\right]= \begin{cases}v_{i j^{\prime}}^{\star}, & \left|\tilde{z}_{j}(t)\right| \leq \tilde{T}_{j} \\
{\left[\underline{v}_{i j}, \bar{v}_{i j}\right],} & \left|\tilde{z}_{j}(t)\right|=\tilde{T}_{j} \\
v_{i j}^{\star \star}, & \left|\tilde{z}_{j}(t)\right|>\tilde{T}_{j},\end{cases} \right. \\
& \mathcal{K}\left[w_{i j}\left(\tilde{z}_{j}(t)\right)\right]=\left\{\begin{array}{ll}
w_{i j}^{\star}, & \left|\tilde{z}_{j}(t)\right| \leq \tilde{T}_{j} \\
{\left[\underline{w}_{i j}, \bar{w}_{i j}\right],} & \left|\tilde{z}_{j}(t)\right|=\tilde{T}_{j} \\
w_{i j}^{\star \star}, & \left|\tilde{z}_{j}(t)\right|>\tilde{T}_{j,}
\end{array} \quad \mathcal{K}\left[x_{i j}\left(\tilde{z}_{j}(t)\right)\right]= \begin{cases}x_{i j}^{\star}, & \left|\tilde{z}_{j}(t)\right| \leq \tilde{T}_{j} \\
{\left[x_{i j}, \bar{x}_{i j}\right],} & \left|\tilde{z}_{j}(t)\right|=\tilde{T}_{j} \\
x_{i j}^{\star \star}, & \left|\tilde{z}_{j}(t)\right|>\tilde{T}_{j} .\end{cases} \right.
\end{aligned}
$$

Then, there exist $\breve{b}_{j i}(t) \in\left[\mathcal{K}\left(b_{j i}\left(\tilde{y}_{i}(t)\right)\right)\right], \check{c}_{j i}(t) \in\left[\mathcal{K}\left(c_{j i}\left(\tilde{y}_{i}(t)\right)\right)\right] \breve{d}_{j i}(t) \in\left[\mathcal{K}\left(d_{j i}\left(\tilde{y}_{i}(t)\right)\right)\right], \check{v}_{i j}(t) \in$ $\left[\mathcal{K}\left(v_{i j}\left(\tilde{z}_{j}(t)\right)\right)\right], \check{w}_{i j}(t) \in\left[\mathcal{K}\left(w_{i j}\left(\tilde{z}_{j}(t)\right)\right)\right]$, and $\check{x}_{i j}(t) \in\left[\mathcal{K}\left(x_{i j}\left(\tilde{z}_{j}(t)\right)\right)\right]$ the measurable function such that

$$
\begin{aligned}
D^{q} \tilde{y}_{i}(t)=-a_{i} \tilde{y}_{i}(t)+\sum_{j=1}^{m} \check{b}_{j i}(t) f_{j}\left(\tilde{z}_{j}(t)\right)+\sum_{j=1}^{m} \check{c}_{j i}(t) f_{j}\left(\tilde{z}_{j}(t-\rho(t))\right)+ & \sum_{j=1}^{m} \check{d}_{j i}(t) \int_{t-r(t)}^{t} f_{j}\left(\tilde{z}_{j}(s)\right) d s \\
& +J_{i}+\zeta_{i}(t), t \geq 0, \\
D^{q} \tilde{z}_{j}(t)=-u_{j} \tilde{z}_{j}(t)+\sum_{i=1}^{n} \check{v}_{i j}(t) g_{i}\left(\tilde{y}_{i}(t)\right)+\sum_{i=1}^{n} \check{w}_{i j}(t) g_{i}\left(\tilde{y}_{i}(t-\rho(t))\right)+ & \sum_{i=1}^{n} \check{x}_{i j}(t) \int_{t-r(t)}^{t} g_{i}\left(\tilde{y}_{i}(s)\right) d s \\
& +H_{j}+\varsigma_{j}(t), t \geq 0 .
\end{aligned}
$$

The initial values of drive-response systems are defined as follows $y_{i}(s)=\hat{\sigma}_{i}(s), z_{j}(s)=$ $\hat{\varrho}_{j}(s), \tilde{y}_{i}(s)=\tilde{\sigma}_{i}(s), \tilde{z}_{j}(s)=\tilde{\varrho}_{j}(s)$, respectively, $s \in[-\omega, 0], \hat{\sigma}_{i}(s), \hat{\varrho}_{j}(s), \tilde{\sigma}_{i}(s), \tilde{\varrho}_{j}(s) \in C([-\omega, 0], R)$, where $\omega=\max \{\rho, r\}$ for $i=1,2, \ldots, n, j=1,2, \ldots, m$. 
Before ending this section, we introduce projective lag synchronization definition, which will play important roles in the proof of our main theorem below.

Definition 4. If there exists a positive constants $\lambda, \tau \in R$, such that for any solutions of FOMBNNs (1) and (4) with different initial values, one can obtain

$$
\lim _{t \rightarrow \infty} \sum_{i=1}^{n}\left|\tilde{y}_{i}(t)-\lambda y_{i}(t-\tau)\right|+\lim _{t \rightarrow \infty} \sum_{j=1}^{m}|| \tilde{z}_{j}(t)-\lambda z_{j}(t-\tau) \mid=0
$$

then (1) and (4) can realize global projective lag synchronization, where $\lambda$ is the projective coefficient and $\tau$ is the projective transmittal delay.

Remark 1. If $\tau=0$, systems (1) and (4) can realize global projective synchronization. If $\tau=0, \lambda=1$, the (1) and (4) can realize global complete asymptotical synchronization. if $\tau=0, \lambda=-1$, systems (1) and (4) can realize anti-synchronization.

Remark 2. If $\lambda=0$, the inequality in above Definition 4 is reduced into the following form:

$$
\lim _{t \rightarrow \infty} \sum_{i=1}^{n}\left|\tilde{y}_{i}(t)\right|+\lim _{t \rightarrow \infty} \sum_{j=1}^{m}\left|\tilde{z}_{j}(t)\right|=0
$$

In this situation, the FOMBNNs (1) is said to be global asymptotical stabilized to the origin.

Assumption $(\mathcal{H})$. The nonlinear activation function $f_{j}$ and $g_{i}$ satisfies the Lipschitz-continuous if there exists constants $L_{i}>0$ and $\tilde{L}_{j}>0$ such that

$$
\begin{aligned}
\left|g_{i}(\tilde{y})-g_{i}(y)\right| & \leq L_{i}|\tilde{y}-y| \\
\left|f_{j}(\tilde{z})-f_{j}(z)\right| & \leq \tilde{L}_{j}|\tilde{z}-z|,
\end{aligned}
$$

for all $y, z, \tilde{y}, \tilde{z} \in R$ and $i=1,2, \ldots, n, j=1,2, \ldots, m$.

\section{Main Results}

In this section, by using the suitable Lyapunov-function, some sufficient conditions are derived to ensure the global projective lag synchronization via hybrid control schemes, and we will derive to guarantee the several synchronization criteria for things such as drive-response systems.

Let $\alpha_{i}(t)=\tilde{y}_{i}(t)-\lambda y_{i}(t-\tau), \beta_{j}(t)=\tilde{z}_{j}(t)-\lambda z_{j}(t-\tau)$ and a hybrid controller is designed as

$$
\zeta_{i}(t)=\hat{\zeta}_{i}(t)+\check{\zeta}_{i}(t) \text { and } \zeta_{j}(t)=\hat{\zeta}_{j}(t)+\check{\zeta}_{j}(t),
$$


where

$$
\begin{aligned}
\hat{\zeta}_{i}(t)= & -\lambda \sum_{j=1}^{m}\left[b_{j i}\left(\tilde{y}_{i}(t)\right)-b_{j i}\left(y_{i}(t)\right)\right] f_{j}\left(z_{j}(t-\tau)\right)-\sum_{j=1}^{m}\left[b_{j i}\left(\tilde{y}_{i}(t)\right)\right]\left[\left(f_{j}\left(\lambda z_{j}(t-\tau)\right)-\lambda\left(f_{j} z_{j}(t-\tau)\right)\right]\right. \\
& -\lambda \sum_{j=1}^{m}\left[c_{j i}\left(\tilde{y}_{i}(t)\right)-c_{j i}\left(y_{i}(t)\right)\right] f_{j}\left(z_{j}(t-\tau-\rho(t-\tau))\right)-\sum_{j=1}^{m}\left[c_{j i}\left(\tilde{y}_{i}(t)\right)\right]\left[f _ { j } \left(\lambda z_{j}(t-\tau\right.\right. \\
& -\rho(t-\tau)))-\lambda\left(f_{j}\left(z_{j}(t-\tau-\rho(t-\tau))\right)\right]-\lambda \sum_{j=1}^{m}\left[d_{j i}\left(\tilde{y}_{i}(t)\right)-d_{j i}\left(y_{i}(t)\right)\right] \int_{t-r(t)}^{t} f_{j}\left(z_{j}(s-\tau)\right) d s \\
& -\sum_{j=1}^{m}\left[d_{j i}\left(\tilde{y}_{i}(t)\right)\right] \int_{t-r(t)}^{t}\left[f_{j}\left(\lambda z_{j}(s-\tau)-\lambda\left(f_{j} z_{j}(s-\tau)\right)\right] d s+(\lambda-1) J_{i},\right. \\
\check{\zeta}_{i}(t)= & -\eta_{i}(t) \alpha_{i}(t), \dot{\eta}_{i}(t)=\frac{-\kappa_{i} \theta_{i}\left|\alpha_{i}(t)\right|}{\eta_{i}(t)}+\kappa_{i}\left|\alpha_{i}(t)\right|
\end{aligned}
$$

and

$$
\begin{aligned}
\hat{\zeta}_{j}(t)= & -\lambda \sum_{i=1}^{n}\left[v_{i j}\left(\tilde{z}_{j}(t)\right)-v_{i j}\left(z_{j}(t)\right)\right] g_{i}\left(y_{i}(t-\tau)\right)-\sum_{i=1}^{n}\left[v_{i j}\left(\tilde{z}_{j}(t)\right)\right]\left[g_{i}\left(\lambda y_{i}(t-\tau)\right)-\lambda\left(g_{i} y_{i}(t-\tau)\right)\right] \\
& -\lambda \sum_{i=1}^{n}\left[w_{i j}\left(\tilde{z}_{j}(t)\right)-w_{i j}\left(z_{j}(t)\right)\right] g_{i}\left(y_{i}(t-\tau-\rho(t-\tau))\right)-\sum_{i=1}^{n}\left[w_{i j}\left(\tilde{z}_{j}(t)\right)\right]\left[g _ { i } \left(\lambda y_{i}(t-\tau\right.\right. \\
& -\rho(t-\tau)))-\lambda\left(g_{i}\left(y_{i}(t-\tau-\rho(t-\tau))\right)\right]-\lambda \sum_{i=1}^{n}\left[x_{i j}\left(\tilde{z}_{j}(t)\right)-x_{i j}\left(z_{j}(t)\right)\right] \int_{t-r(t)}^{t} g_{i}\left(y_{i}(s-\tau)\right) d s \\
& -\sum_{i=1}^{n}\left[x_{i j}\left(\tilde{z}_{j}(t)\right)\right] \int_{t-r(t)}^{t}\left[g_{i}\left(\lambda y_{i}(s-\tau)\right)-\lambda\left(g_{i} y_{i}(s-\tau)\right)\right] d s+(\lambda-1) H_{j}, \\
\check{\zeta}_{j}(t)= & -\mu_{j}(t) \beta_{j}(t), \dot{\mu}_{j}(t)=\frac{-\iota_{j} \delta_{j}\left|\beta_{j}(t)\right|}{\mu_{j}(t)}+\iota_{j}\left|\beta_{j}(t)\right|,
\end{aligned}
$$

where $\kappa_{i}, \theta_{i}, \xi_{i}, \iota_{j}, \delta_{j}$ and $\gamma_{j}$ are the positive constants, $\eta_{i}(t)$ and $\mu_{j}(t)$ stands for the adaptive coupling strengths. Based on the hybrid controller scheme (7), combining Equations (2) and (5), then the synchronization error system can be obtained as follows:

$$
\begin{aligned}
D^{q} \alpha_{i}(t) \in & -a_{i} \alpha_{i}(t)+\sum_{j=1}^{m} \mathcal{K}\left[b_{j i}\left(\alpha_{i}(t)+\lambda y_{i}(t-\tau)\right)\right]\left[f_{j}\left(\beta_{j}(t)+\lambda z_{j}(t-\tau)\right)-f_{j}\left(\lambda z_{j}(t-\tau)\right)\right] \\
& +\sum_{j=1}^{m} \mathcal{K}\left[c_{j i}\left(\alpha_{i}(t)+\lambda y_{i}(t-\tau)\right)\right]\left[f_{j}\left(\beta_{j}(t-\rho(t))+\lambda z_{j}(t-\tau-\rho(t-\tau))\right)-f_{j}\left(\lambda z_{j}(t-\tau\right.\right. \\
& -\rho(t-\tau)))]+\sum_{j=1}^{m} \mathcal{K}\left[d_{j i}\left(\alpha_{i}(t)+\lambda y_{i}(t-\tau)\right)\right] \int_{t-r(t)}^{t}\left[f_{j}\left(\beta_{j}(s)+\lambda z_{j}(s-\tau)\right)\right. \\
& \left.-f_{j}\left(\lambda z_{j}(s-\tau)\right)\right] d s-\eta_{i}(t) \alpha_{i}(t), t \geq 0, \\
D^{q} \beta_{j}(t) \in \quad & -u_{j} \tilde{z}_{j}(t)+\sum_{i=1}^{n} \mathcal{K}\left[v_{i j}\left(\beta_{j}(t)+\lambda z_{j}(t-\tau)\right)\right]\left[g_{i}\left(\alpha_{i}(t)+\lambda y_{i}(t-\tau)\right)-g_{i}\left(\lambda y_{i}(t-\tau)\right)\right] \\
& +\sum_{i=1}^{n} \mathcal{K}\left[w_{i j}\left(\beta_{j}(t)+\lambda z_{j}(t-\tau)\right)\right]\left[g_{i}\left(\alpha_{i}(t-\rho(t))+\lambda y_{i}(t-\tau-\rho(t-\tau))\right)-g_{i}\left(\lambda y_{i}(t-\tau\right.\right. \\
& -\rho(t-\tau)))]+\sum_{i=1}^{n} \mathcal{K}\left[x_{i j}\left(\beta_{j}(t)+\lambda z_{j}(t-\tau)\right)\right] \int_{t-r(t)}^{t}\left[g_{i}\left(\alpha_{i}(s)+\lambda y_{i}(s-\tau)\right)\right. \\
& \left.-g_{i}\left(\lambda y_{i}(s-\tau)\right)\right] d s-\mu_{j}(t) \beta_{j}(t), t \geq 0 .
\end{aligned}
$$


or $\breve{b}_{j i}\left(\alpha_{i}(t)+\lambda y_{i}(t-\tau)\right) \in \mathcal{K}\left[b_{j i}\left(\alpha_{i}(t)+\lambda y_{i}(t-\tau)\right)\right], \check{c}_{j i}\left(\alpha_{i}(t)+\lambda y_{i}(t-\tau)\right) \in \mathcal{K}\left[c_{j i}\left(\alpha_{i}(t)+\lambda y_{i}(t-\right.\right.$ $\tau))], \breve{d}_{j i}\left(\alpha_{i}(t)+\lambda y_{i}(t-\tau)\right) \in \mathcal{K}\left[d_{i i}\left(\alpha_{i}(t)+\lambda y_{i}(t-\tau)\right)\right], \check{v}_{i j}\left(\beta_{j}(t)+\lambda z_{j}(t-\tau)\right) \in \mathcal{K}\left[v_{i j}\left(\beta_{j}(t)+\lambda z_{j}(t-\right.\right.$ $\tau))], \breve{w}_{i j}\left(\beta_{j}(t)+\lambda z_{j}(t-\tau)\right) \in \mathcal{K}\left[w_{i j}\left(\beta_{j}(t)+\lambda z_{j}(t-\tau)\right)\right]$ and $\check{x}_{i j}\left(\beta_{j}(t)+\lambda z_{j}(t-\tau)\right) \in \mathcal{K}\left[x_{i j}\left(\beta_{j}(t)+\right.\right.$ $\left.\left.\lambda z_{j}(t-\tau)\right)\right]$ exist such that

$$
\begin{aligned}
D^{q} \alpha_{i}(t)= & -\left(a_{i}+\eta_{i}(t)\right) \alpha_{i}(t)+\sum_{j=1}^{m} \breve{b}_{j i}\left[\left(\alpha_{i}(t)+\lambda y_{i}(t-\tau)\right)\right]\left[f_{j}\left(\beta_{j}(t)+\lambda z_{j}(t-\tau)\right)-f_{j}\left(\lambda z_{j}(t-\tau)\right)\right] \\
& +\sum_{j=1}^{m} \check{c}_{j i}\left[\left(\alpha_{i}(t)+\lambda y_{i}(t-\tau)\right)\right]\left[f_{j}\left(\beta_{j}(t-\rho(t))+\lambda z_{j}(t-\tau-\rho(t-\tau))\right)-f_{j}\left(\lambda z_{j}(t-\tau-\rho(t\right.\right. \\
& -\tau)))]+\sum_{j=1}^{m} \breve{d}_{j i}\left[\left(\alpha_{i}(t)+\lambda y_{i}(t-\tau)\right)\right] \int_{t-r(t)}^{t}\left[f_{j}\left(\beta_{j}(s)+\lambda z_{j}(s-\tau)\right)-f_{j}\left(\lambda z_{j}(s-\tau)\right)\right] d s
\end{aligned}
$$

and

$$
\begin{aligned}
D^{q} \beta_{j}(t)= & -\left(u_{j}+\mu_{j}(t)\right) \beta_{j}(t)+\sum_{i=1}^{n} \check{v}_{i j}\left[\left(\beta_{j}(t)+\lambda z_{j}(t-\tau)\right)\right]\left[g_{i}\left(\alpha_{i}(t)+\lambda y_{i}(t-\tau)\right)-g_{i}\left(\lambda y_{i}(t-\tau)\right)\right] \\
& +\sum_{i=1}^{n} \check{w}_{i j}\left[\left(\beta_{j}(t)+\lambda z_{j}(t-\tau)\right)\right]\left[g_{i}\left(\alpha_{i}(t-\rho(t))+\lambda y_{i}(t-\tau-\rho(t-\tau))\right)-g_{i}\left(\lambda y_{i}(t-\tau-\rho(t\right.\right. \\
& -\tau)))]+\sum_{i=1}^{n} \check{x}_{i j}\left[\left(\beta_{j}(t)+\lambda z_{j}(t-\tau)\right)\right] \int_{t-r(t)}^{t}\left[g_{i}\left(\alpha_{i}(s)+\lambda y_{i}(s-\tau)\right)-g_{i}\left(\lambda y_{i}(s-\tau)\right)\right] d s .
\end{aligned}
$$

Theorem 1. Suppose that Assumptions $[\mathcal{H}]$ hold, then drive system (1) and response system (4) are globally projective lag synchronized via hybrid controller (7) if the following conditions hold:

$$
\begin{aligned}
& \Omega_{1}=\min _{1 \leq i \leq n}\left\{a_{i}+\theta_{i}-\sum_{j=1}^{m}\left(\mathbb{V}_{i j}+\frac{\mathbb{W}_{i j}}{1-\sigma}+\rho \mathbb{X}_{i j}\right) L_{i}\right\}>0, \\
& \Theta_{2}=\min _{1 \leq j \leq m}\left\{u_{j}+\delta_{j}-\sum_{i=1}^{n}\left(\mathbb{B}_{j i}+\frac{\mathbb{C}_{j i}}{1-\sigma}+\rho \mathbb{D}_{j i}\right) \tilde{L}_{j}\right\}>0 .
\end{aligned}
$$

Here, $\mathbb{B}_{j i}=\max \left\{\left|b_{j i}^{\star}\right|,\left|b_{j i}^{\star \star}\right|\right\}, \quad \mathbb{C}_{j i}=\max \left\{\left|c_{j i}^{\star}\right|,\left|c_{j i}^{\star \star}\right|\right\}, \quad \mathbb{D}_{j i}=\max \left\{\left|d_{j i}^{\star}\right|,\left|d_{j i}^{\star \star}\right|\right\}, \quad \mathbb{V}_{i j}=$ $\max \left\{\left|v_{i j}^{\star}\right|,\left|v_{i j}^{\star \star}\right|\right\}, \mathbb{W}_{i j}=\max \left\{\left|w_{i j}^{\star}\right|,\left|w_{i j}^{\star \star}\right|\right\}$ and $\mathbb{X}_{i j}=\max \left\{\left|x_{i j}^{\star}\right|,\left|x_{i j}^{\star \star}\right|\right\}$, while $\theta_{i}>0$ and $\delta_{j}>0$ denote constants.

Proof. Consider the following Lyapunov-like functional

$$
V(t)=V_{1}(t)+V_{2}(t)+V_{3}(t)
$$

where

$$
\begin{aligned}
V_{1}(t)= & D^{-(1-q)}\left(\sum_{i=1}^{n}\left|\alpha_{i}(t)\right|+\sum_{j=1}^{m}\left|\beta_{j}(t)\right|\right), \\
V_{2}(t)= & \sum_{i=1}^{n} \frac{1}{2 \kappa_{i}} \eta_{i}(t)^{2}+\sum_{j=1}^{m} \frac{1}{2 \iota_{j}} \mu_{j}(t)^{2}, \\
V_{3}(t)= & \sum_{j=1}^{m} \sum_{i=1}^{n} \mathbb{C}_{j i} \tilde{L}_{j} \int_{t-\tau(t)}^{t}\left|\beta_{j}(s)\right| \mathrm{d} s+\sum_{j=1}^{m} \sum_{i=1}^{n} \mathbb{D}_{j i} \tilde{L}_{j} \int_{-\rho(t)}^{0} \int_{t+s}^{t}\left|\beta_{j}(s)\right| \mathrm{d} s \\
& +\sum_{i=1}^{n} \sum_{j=1}^{m} \mathbb{W}_{i j} L_{i} \int_{t-\tau(t)}^{t}\left|\alpha_{i}(s)\right| \mathrm{d} s+\sum_{i=1}^{n} \sum_{j=1}^{m} \mathbb{X}_{i j} L_{i} \int_{-\rho(t)}^{0} \int_{t+s}^{t}\left|\alpha_{i}(s)\right| \mathrm{d} s .
\end{aligned}
$$


Then, by applying the Riemann-Liouville derivative for Lyapunov functional (10), one has

$$
\dot{V}(t)=\dot{V}_{1}(t)+\dot{V}_{2}(t)+\dot{V}_{3}(t)
$$

where

$$
\begin{aligned}
& \dot{V}_{1}(t)=D^{q}\left[\sum_{i=1}^{n}\left|\alpha_{i}(t)\right|+\sum_{j=1}^{m}\left|\beta_{j}(t)\right|\right] \\
& \leq \sum_{i=1}^{n} \operatorname{sgn}\left(\alpha_{i}(t)\right) D^{q} \alpha_{i}(t)+\sum_{j=1}^{m} \operatorname{sgn}\left(\beta_{j}(t)\right) D^{q} \beta_{j}(t) \\
& \leq \sum_{i=1}^{n} \operatorname{sgn}\left(\alpha_{i}(t)\right)\left\{-\left(a_{i}+\eta_{i}(t)\right) \alpha_{i}(t)+\sum_{j=1}^{m} \check{b}_{j i}\left[\left(\alpha_{i}(t)+\lambda y_{i}(t-\tau)\right)\right]\left[f_{j}\left(\beta_{j}(t)+\lambda z_{j}(t-\tau)\right)\right.\right. \\
& \left.-f_{j}\left(\lambda z_{j}(t-\tau)\right)\right]+\sum_{j=1}^{m} \check{c}_{j i}\left[\left(\alpha_{i}(t)+\lambda y_{i}(t-\tau)\right)\right]\left[f_{j}\left(\beta_{j}(t-\rho(t))+\lambda z_{j}(t-\tau-\rho(t-\tau))\right)\right. \\
& \left.-f_{j}\left(\lambda z_{j}(t-\tau-\rho(t-\tau))\right)\right]+\sum_{j=1}^{m} \check{d}_{j i}\left[\left(\alpha_{i}(t)+\lambda y_{i}(t-\tau)\right)\right] \int_{t-r(t)}^{t}\left[f_{j}\left(\beta_{j}(s)+\lambda z_{j}(s-\tau)\right)\right. \\
& \left.\left.-f_{j}\left(\lambda z_{j}(s-\tau)\right)\right] d s\right\}+\sum_{j=1}^{m} \operatorname{sgn}\left(\beta_{j}(t)\right)\left\{-\left(u_{j}+\mu_{j}(t)\right) \beta_{j}(t)+\sum_{i=1}^{n} \check{v}_{i j}\left[\left(\beta_{j}(t)+\lambda z_{j}(t-\tau)\right)\right]\right. \\
& {\left[g_{i}\left(\alpha_{i}(t)+\lambda y_{i}(t-\tau)\right)-g_{i}\left(\lambda y_{i}(t-\tau)\right)\right]+\sum_{i=1}^{n} \check{w}_{i j}\left[\left(\beta_{j}(t)+\lambda z_{j}(t-\tau)\right)\right]\left[g _ { i } \left(\alpha_{i}(t-\rho(t))\right.\right.} \\
& \left.\left.+\lambda y_{i}(t-\tau-\rho(t-\tau))\right)-g_{i}\left(\lambda y_{i}(t-\tau-\rho(t-\tau))\right)\right]+\sum_{i=1}^{n} \check{x}_{i j}\left[\left(\beta_{j}(t)+\lambda z_{j}(t-\tau)\right)\right] \\
& \left.\int_{t-r(t)}^{t}\left[g_{i}\left(\alpha_{i}(s)+\lambda y_{i}(s-\tau)\right)-g_{i}\left(\lambda y_{i}(s-\tau)\right)\right] d s\right\} \\
& \leq \sum_{i=1}^{n}\left\{-\left(a_{i}+\eta_{i}(t)\right)\left|\alpha_{i}(t)\right|+\sum_{j=1}^{m} \mathbb{B}_{j i} \tilde{L}_{j}\left|\beta_{j}(t)\right|+\sum_{j=1}^{m} \mathbb{C}_{j i} \tilde{L}_{j}\left|\beta_{j}(t-\rho(t))\right|\right. \\
& \left.+\sum_{j=1}^{m} \mathbb{D}_{j i} \tilde{L}_{j} \int_{t-r(t)}^{t}\left|\beta_{j}(s)\right| d s\right\}+\sum_{j=1}^{m}\left\{-\left(u_{j}+\mu_{j}(t)\right)\left|\beta_{j}(t)\right|+\sum_{i=1}^{n} \mathbb{V}_{i j} L_{i}\left|\alpha_{i}(t)\right|\right. \\
& \left.+\sum_{i=1}^{n} \mathbb{W}_{i j} L_{i}\left|\alpha_{i}(t-\rho(t))\right|+\sum_{i=1}^{n} \mathbb{X}_{i j} L_{i} \int_{t-r(t)}^{t}\left|\alpha_{i}(s)\right| d s\right\} \\
& \dot{V}_{2}(t)=\sum_{i=1}^{n} \frac{2 \eta_{i}(t) \dot{\eta}_{i}(t)}{2 \kappa_{i}}+\sum_{j=1}^{m} \frac{2 \mu_{j}(t) \dot{\mu}_{j}(t)}{2 \iota_{j}} \\
& =\sum_{i=1}^{n} \frac{\eta_{i}(t)}{\kappa_{i}}\left[\frac{-\kappa_{i} \theta_{i}\left|\alpha_{i}(t)\right|}{\eta_{i}(t)}+\kappa_{i}\left|\alpha_{i}(t)\right|\right]+\sum_{j=1}^{m} \frac{\mu_{j}(t)}{\iota_{j}}\left[\frac{-\iota_{j} \delta_{j}\left|\beta_{j}(t)\right|}{\mu_{j}(t)}+\iota_{j}\left|\beta_{j}(t)\right|\right] \\
& =\sum_{i=1}^{n}\left[-\theta_{i}\left|\alpha_{i}(t)\right|+\eta_{i}(t)\left|\alpha_{i}(t)\right|\right]+\sum_{j=1}^{m}\left[-\delta_{j}\left|\beta_{j}(t)\right|+\mu_{j}(t)\left|\beta_{j}(t)\right|\right],
\end{aligned}
$$


and

$$
\begin{aligned}
\dot{V}_{3}(t)= & -\frac{1}{1-\sigma} \sum_{i=1}^{n} \sum_{j=1}^{m} \mathbb{W}_{i j} L_{i}\left[(1-\dot{\rho}(t))\left|\alpha_{i}(t-\rho(t))\right|-\left|\alpha_{i}(t)\right|\right]+\sum_{i=1}^{n} \sum_{j=1}^{m} \mathbb{X}_{i j} L_{i} \int_{-r(t)}^{0}\left[\left|\alpha_{i}(t)\right|\right. \\
& \left.-\left|\alpha_{i}(t+s)\right|\right] d s-\frac{1}{1-\sigma} \sum_{j=1}^{m} \sum_{i=1}^{n} \mathbb{C}_{j i} \tilde{L}_{j}\left[(1-\dot{\rho}(t))\left|\beta_{j}(t-\rho(t))\right|-\left|\beta_{j}(t)\right|\right] \\
& +\sum_{j=1}^{m} \sum_{i=1}^{n} \mathbb{D}_{j i} \tilde{L}_{j} \int_{-r(t)}^{0}\left[\left|\beta_{j}(t)\right|-\left|\beta_{j}(t+s)\right|\right] d s \\
\leq & -\sum_{i=1}^{n} \sum_{j=1}^{m} \mathbb{W}_{i j} L_{i}\left|\alpha_{i}(t-\rho(t))\right|+\frac{1}{1-\sigma} \sum_{i=1}^{n} \sum_{j=1}^{m} \mathbb{W}_{i j} L_{i}\left|\alpha_{i}(t)\right|+\sum_{i=1}^{n} \sum_{j=1}^{m} \mathbb{X}_{i j} L_{i} r\left|\alpha_{i}(t)\right| \\
& -\sum_{i=1}^{n} \sum_{j=1}^{m} \mathbb{X}_{i j} L_{i} \int_{t-r(t)}^{t}\left|\alpha_{i}(s)\right| d s-\sum_{j=1}^{m} \sum_{i=1}^{n} \mathbb{C}_{j i} \tilde{L}_{j}\left|\beta_{j}(t-\rho(t))\right|+\frac{1}{1-\sigma} \sum_{j=1}^{m} \sum_{i=1}^{n} \mathbb{C}_{j i} \tilde{L}_{j}\left|\beta_{j}(t)\right| \\
& +\sum_{j=1}^{m} \sum_{i=1}^{n} \mathbb{D}_{j i} \tilde{L}_{j} r\left|\beta_{j}(t)\right|-\sum_{j=1}^{m} \sum_{i=1}^{n} \mathbb{D}_{j i} \tilde{L}_{j} \int_{t-r(t)}^{t}\left|\beta_{j}(s)\right| d s .
\end{aligned}
$$

From Equation (11), it follows that

$$
\begin{aligned}
\dot{V}(t) \leq & -\sum_{i=1}^{n}\left\{a_{i}+\theta_{i}-\sum_{j=1}^{m}\left(\mathbb{V}_{i j}+\frac{\mathbb{W}_{i j}}{1-\sigma}+r \mathbb{X}_{i j}\right) L_{i}\right\}\left|\alpha_{i}(t)\right| \\
& -\sum_{j=1}^{m}\left\{u_{j}+\delta_{j}-\sum_{i=1}^{n}\left(\mathbb{B}_{j i}+\frac{\mathbb{C}_{j i}}{1-\sigma}+r \mathbb{D}_{j i}\right) \tilde{L}_{j}\right\}\left|\beta_{j}(t)\right| .
\end{aligned}
$$

We can choose the appropriate positive scalars $\Omega_{1}, \Theta_{1}$ such that

$$
\begin{aligned}
\dot{V}(t) & \leq-\Omega_{1} \sum_{i=1}^{n}\left|\alpha_{i}(t)\right|-\Theta_{1} \sum_{j=1}^{m}\left|\beta_{j}(t)\right| \\
& \leq-Y\left[\sum_{i=1}^{n}\left|\alpha_{i}(t)\right|+\sum_{j=1}^{m}\left|\beta_{j}(t)\right|\right] \\
& \leq-Y \Psi(t),
\end{aligned}
$$

where $\Psi(t)=\sum_{i=1}^{n}\left|\alpha_{i}(t)\right|+\sum_{j=1}^{m}\left|\beta_{j}(t)\right|$ and $Y=\min \left\{\Omega_{1}, \Theta_{1}\right\}$. We can get $V(0) \geq V(t)+$ $Y \int_{0}^{t} \Psi(\gamma) d \gamma$; it is simple to get $\int_{0}^{t} \Psi(\gamma) d \gamma$ has a finite limit and $\Psi(\gamma)$ bounded that is $\alpha_{i}(t)$ and $\beta_{j}(t)$ is bounded. Therefore, there exist a constant $\mathcal{\omega}>0$ such that $D^{q}|\Psi(t)| \leq \mathcal{\omega}, t \geq 0$ is bounded. Based on the Barbalet's Lemma 2, we show that $\Psi(t)$ is uniformly continuous. For $0 \leq t_{1}<t_{2}$ 


$$
\begin{aligned}
\left.\mid \Psi\left(t_{1}\right)-\Psi\left(t_{2}\right)\right) \mid= & \left|\left(\Psi\left(t_{1}\right)-\Psi(0)\right)-\left(\Psi\left(t_{2}\right)-\Psi(0)\right)\right| \\
= & \left|D^{-q} D^{q}\left(\Psi\left(t_{1}\right)\right)-D^{-q} D^{q}\left(\Psi\left(t_{2}\right)\right)\right| \\
= & \frac{1}{\Gamma(q)}\left|\int_{0}^{t_{1}}\left(t_{1}-\gamma\right)^{q-1} D^{q}(\Psi(\gamma)) d \gamma-\int_{0}^{t_{2}}\left(t_{2}-\gamma\right)^{q-1} D^{q}(\Psi(\gamma)) d \gamma\right| \\
\leq & \frac{1}{\Gamma(q)}\left[\left|\int_{0}^{t_{1}}\left[\left(t_{1}-\gamma\right)^{q-1}-\left(t_{2}-\gamma\right)^{q-1}\right] D^{q}(\Psi(\gamma)) d \gamma\right|\right. \\
& \left.+\left|\int_{t_{1}}^{t_{2}}\left(t_{2}-\gamma\right)^{q-1} D^{q}(\Psi(\gamma)) d \gamma\right|\right] \\
\leq & \frac{\omega}{\Gamma(q)}\left[\left|\int_{0}^{t_{1}}\left[\left(t_{1}-\gamma\right)^{q-1}-\left(t_{2}-\gamma\right)^{q-1}\right] d \gamma\right|+\left|\int_{t_{1}}^{t_{2}}\left(t_{2}-\gamma\right)^{q-1} d \gamma\right|\right] \\
\leq & \frac{2 \omega}{q \Gamma(q)}\left[t_{1}^{q}-t_{2}^{q}+2\left(t_{2}-t_{1}\right)^{q}\right] \\
\leq & \frac{2 \omega}{\Gamma(q+1)}\left(t_{2}-t_{1}\right)^{q}<\varepsilon
\end{aligned}
$$

where $\left|t_{2}-t_{1}\right|<\varepsilon(\iota)=\sqrt[q]{\frac{l \Gamma(q+1)}{2 \omega}}$. Thus, $\Psi(t)$ is uniformly continuous, by Lemma 2 , we have

$$
\lim _{t \rightarrow+\infty} \sum_{i=1}^{n}\left|\alpha_{i}(t)\right|=0
$$

and

$$
\lim _{t \rightarrow+\infty} \sum_{j=1}^{m}\left|\beta_{j}(t)\right|=0
$$

Therefore, based on Definition 4, systems (1) and (4) are globally projective lag synchronized via hybrid controller (7). The proof is completed.

Suppose the systems (1) and (4) are without distributed delay, then we have to select the following hybrid controller in a response system such as

$$
\zeta_{i}(t)=\hat{\zeta}_{i}(t)+\breve{\zeta}_{i}(t) \text { and } \zeta_{j}(t)=\hat{\zeta}_{j}(t)+\breve{\zeta}_{j}(t),
$$


where

$$
\begin{aligned}
\hat{\zeta}_{i}(t)= & -\lambda \sum_{j=1}^{m}\left[b_{j i}\left(\tilde{y}_{i}(t)\right)-b_{j i}\left(y_{i}(t)\right)\right] f_{j}\left(z_{j}(t-\tau)\right)-\sum_{j=1}^{m}\left[b_{j i}\left(\tilde{y}_{i}(t)\right)\right]\left[\left(f_{j}\left(\lambda z_{j}(t-\tau)\right)-\lambda\left(f_{j}\left(z_{j}(t-\tau)\right)\right)\right]\right. \\
& -\lambda \sum_{j=1}^{m}\left[c_{j i}\left(\tilde{y}_{i}(t)\right)-c_{j i}\left(y_{i}(t)\right)\right] f_{j}\left(z_{j}(t-\tau-\rho(t-\tau))\right) \\
& -\sum_{j=1}^{m}\left[c_{j i}\left(\tilde{y}_{i}(t)\right)\right]\left[f_{j}\left(\lambda z_{j}(t-\tau-\rho(t-\tau))\right)-\lambda\left(f_{j}\left(z_{j}(t-\tau-\rho(t-\tau))\right)\right]+(\lambda-1) J_{i}\right. \\
\check{\zeta}_{i}(t)= & -\eta_{i}(t) \alpha_{i}(t), \dot{\eta}_{i}(t)=\frac{-\kappa_{i} \theta_{i}\left|\alpha_{i}(t)\right|}{\eta_{i}(t)}+\kappa_{i}\left|\alpha_{i}(t)\right|
\end{aligned}
$$

and

$$
\begin{aligned}
\hat{\zeta}_{j}(t)= & -\lambda \sum_{i=1}^{n}\left[v_{i j}\left(\tilde{z}_{j}(t)\right)-v_{i j}\left(z_{j}(t)\right)\right] g_{i}\left(y_{i}(t-\tau)\right)-\sum_{i=1}^{n}\left[v_{i j}\left(\tilde{z}_{j}(t)\right)\right]\left[g_{i}\left(\lambda y_{i}(t-\tau)\right)-\lambda\left(g_{i}\left(y_{i}(t-\tau)\right)\right]\right. \\
& -\lambda \sum_{i=1}^{n}\left[w_{i j}\left(\tilde{z}_{j}(t)\right)-w_{i j}\left(z_{j}(t)\right)\right] g_{i}\left(y_{i}(t-\tau-\rho(t-\tau))\right) \\
& -\sum_{i=1}^{n}\left[w_{i j}\left(\tilde{z}_{j}(t)\right)\right]\left[g_{i}\left(\lambda y_{i}(t-\tau-\rho(t-\tau))\right)-\lambda\left(g_{i}\left(y_{i}(t-\tau-\rho(t-\tau))\right)\right]+(\lambda-1) H_{j}\right. \\
\check{\zeta}_{j}(t)= & -\mu_{j}(t) \beta_{j}(t), \dot{\mu}_{j}(t)=\frac{-\iota_{j} \delta_{j}\left|\beta_{j}(t)\right|}{\mu_{j}(t)}+\iota_{j}\left|\beta_{j}(t)\right| .
\end{aligned}
$$

These corollaries are directly obtained from our main Theorem 3.

Corollary 1. Suppose that Assumptions [H] hold, then drive system (1) and response system (4) without distributed delay are globally projective lag synchronized via hybrid controller (12), if the following conditions hold:

$$
\begin{aligned}
& \Omega_{1}=\min _{1 \leq i \leq n}\left\{a_{i}+\theta_{i}-\sum_{j=1}^{m}\left(\mathbb{V}_{i j}+\frac{\mathbb{W}_{i j}}{1-\sigma}\right) L_{i}\right\}>0, \\
& \Theta_{2}=\min _{1 \leq j \leq m}\left\{u_{j}+\delta_{j}-\sum_{i=1}^{n}\left(\mathbb{B}_{j i}+\frac{\mathbb{C}_{i j}}{1-\sigma}\right) \tilde{L}_{j}\right\}>0,
\end{aligned}
$$

where $\mathbb{B}_{j i}=\max \left\{\left|b_{j i}^{\star}\right|,\left|b_{j i}^{\star \star}\right|\right\}, \quad \mathbb{C}_{j i}=\max \left\{\left|c_{j i}^{\star}\right|,\left|c_{j i}^{\star \star}\right|\right\}, \quad \mathbb{V}_{i j}=\max \left\{\left|v_{i j}^{\star}\right|,\left|v_{i j}^{\star \star}\right|\right\}$ and $\mathbb{W}_{i j}=$ $\max \left\{\left|w_{i j}^{\star}\right|,\left|w_{i j}^{\star \star}\right|\right\}, \theta_{i}, \xi_{i}, \delta_{j}$ and $\gamma_{j}$ denotes the appropriate positive constants.

Proof. The proof is repeated to Theorem 3. Hence, the proof is omitted here.

Supposing the drive-response systems are without discrete and distributed delay, then we have to select the following hybrid controller in a response system such as

$$
\zeta_{i}(t)=\hat{\zeta}_{i}(t)+\check{\zeta}_{i}(t) \text { and } \zeta_{j}(t)=\hat{\zeta}_{j}(t)+\check{\zeta}_{j}(t),
$$


where

$$
\begin{aligned}
\hat{\zeta}_{i}(t) & =-\lambda \sum_{j=1}^{m}\left[b_{j i}\left(\tilde{y}_{i}(t)\right)-b_{j i}\left(y_{i}(t)\right)\right] f_{j}\left(z_{j}(t)\right)-\sum_{j=1}^{m}\left[b_{j i}\left(\tilde{y}_{i}(t)\right)\right]\left[\left(f_{j}\left(\lambda z_{j}(t)\right)-\lambda\left(f_{j} z_{j}(t)\right)\right]+(\lambda-1) J_{i}\right. \\
\check{\zeta}_{i}(t) & =-\eta_{i}(t) \alpha_{i}(t), \dot{\eta}_{i}(t)=\frac{-\kappa_{i} \theta_{i}\left|\alpha_{i}(t)\right|}{\eta_{i}(t)}+\kappa_{i}\left|\alpha_{i}(t)\right|, \\
\hat{\zeta}_{j}(t) & =-\lambda \sum_{i=1}^{n}\left[v_{i j}\left(\tilde{z}_{j}(t)\right)-v_{i j}\left(z_{j}(t)\right)(t)\right] g_{i}\left(y_{i}(t)\right)-\sum_{i=1}^{n}\left[v_{i j}\left(\tilde{z}_{j}(t)\right)\right]\left[g_{i}\left(\lambda y_{i}(t)\right)-\lambda\left(g_{i} y_{i}(t)\right)\right]+(\lambda-1) H_{j}, \\
\check{\zeta}_{j}(t) & =-\mu_{j}(t) \beta_{j}(t), \dot{\mu}_{j}(t)=\frac{-\iota_{j} \delta_{j}\left|\beta_{j}(t)\right|}{\mu_{j}(t)}+\iota_{j}\left|\beta_{j}(t)\right| .
\end{aligned}
$$

These corollaries are directly obtained from our main Theorem 3.

Corollary 2. Suppose that Assumptions $[\mathcal{H}]$ hold, then systems (1) and (4) are without discrete and distributed delay are globally projective lag synchronized via hybrid controller (13) if the following conditions hold:

$$
\begin{aligned}
& \Omega_{1}=\min _{1 \leq i \leq n}\left\{a_{i}+\theta_{i}-\sum_{j=1}^{m} \mathbb{V}_{i j} L_{i}\right\}>0, \\
& \Theta_{2}=\min _{1 \leq j \leq m}\left\{u_{j}+\delta_{j}-\sum_{i=1}^{n} \mathbb{B}_{j i} \tilde{L}_{j}\right\}>0 .
\end{aligned}
$$

where $\mathbb{B}_{j i}=\max \left\{\left|b_{j i}^{\star}\right|,\left|b_{j i}^{\star \star}\right|\right\}$ and $\mathbb{V}_{i j}=\max \left\{\left|v_{i j}^{\star}\right|,\left|v_{i j}^{\star \star}\right|\right\}, \theta_{i}$ and $\delta_{j}$ denotes the appropriate positive constants.

Proof. The proof is similar to the proof of Theorem 3. Hence, the proof is omitted here.

Let $\alpha_{i}(t)=\tilde{y}_{i}(t)-\lambda y_{i}(t)$ and $\beta_{j}(t)=\tilde{z}_{j}(t)-\lambda z_{j}(t)$. If $\tau=0$, the control input (7) can be reduced into the following form:

$$
\begin{aligned}
\zeta_{i}(t)= & -\lambda \sum_{j=1}^{m}\left[b_{j i}\left(\tilde{y}_{i}(t)\right)-b_{j i}\left(y_{i}(t)\right)\right] f_{j}\left(z_{j}(t)\right)-\lambda \sum_{j=1}^{m}\left[c_{j i}\left(\tilde{y}_{i}(t)\right)-c_{j i}\left(y_{i}(t)\right)\right] f_{j}\left(z_{j}(t-\rho(t))\right) \\
& -\lambda \sum_{j=1}^{m}\left[d_{j i}\left(\tilde{y}_{i}(t)\right)-d_{j i}\left(y_{i}(t)\right)\right] \int_{t-r(t)}^{t} f_{j}\left(z_{j}(s)\right) d s-\sum_{j=1}^{m}\left[b_{j i}\left(\tilde{y}_{i}(t)\right)\right]\left[\left(f_{j}\left(\lambda z_{j}(t)\right)-\lambda\left(f_{j}\left(z_{j}(t)\right)\right]\right.\right. \\
& -\lambda \sum_{j=1}^{m}\left[c_{j i}\left(\tilde{y}_{i}(t)\right)\right]\left[f_{j}\left(\lambda z_{j}(t-\rho(t))\right)-\lambda\left(f_{j}\left(z_{j}(t-\rho(t))\right)\right]-\sum_{j=1}^{m}\left[d_{j i}\left(\tilde{y}_{i}(t)\right)\right] \int_{t-r(t)}^{t}\left[f _ { j } \left(\lambda z_{j}(s)\right.\right.\right. \\
& \left.-\lambda\left(f_{j} z_{j}(s)\right)\right] d s+(\lambda-1) J_{i}-\eta_{i}(t) \alpha_{i}(t) \\
\varsigma_{j}(t)= & -\lambda \sum_{i=1}^{n}\left[v_{i j}\left(\tilde{z}_{j}(t)\right)-v_{i j}\left(z_{j}(t)\right)\right] g_{i}\left(y_{i}(t)\right)-\lambda \sum_{i=1}^{n}\left[w_{i j}\left(\tilde{z}_{j}(t)\right)-w_{i j}\left(z_{j}(t)\right)\right] g_{i}\left(y_{i}(t-\rho(t))\right) \\
& -\lambda \sum_{i=1}^{n}\left[x_{i j}\left(\tilde{z}_{j}(t)\right)-x_{i j}\left(z_{j}(t)\right)\right] \int_{t-r(t)}^{t} g_{i}\left(y_{i}(s)\right) d s-\sum_{i=1}^{n}\left[v_{i j}\left(\tilde{z}_{j}(t)\right)\right]\left[g_{i}\left(\lambda y_{i}(t)\right)-\lambda\left(g_{i}\left(y_{i}(t)\right)\right)\right] \\
& -\sum_{i=1}^{n}\left[w_{i j}\left(\tilde{z}_{j}(t)\right)\right]\left[g_{i}\left(\lambda y_{i}(t-\rho(t))\right)-\lambda\left(g_{i}\left(y_{i}(t-\rho(t))\right)\right)\right]-\sum_{i=1}^{n}\left[x_{i j}\left(\tilde{z}_{j}(t)\right)\right] \int_{t-r(t)}^{t}\left[g_{i}\left(\lambda y_{i}(s)\right)\right. \\
& \left.-\lambda\left(g_{i} y_{i}(s)\right)\right] d s+(\lambda-1) H_{j}-\mu_{j}(t) \beta_{j}(t) .
\end{aligned}
$$


Let $\alpha_{i}(t)=\tilde{y}_{i}(t)-y_{i}(t)$ and $\beta_{j}(t)=\tilde{z}_{j}(t)-z_{j}(t)$. If $\tau=0$ and $\lambda=1$, the control input (7) can be reduced into the following form:

$$
\begin{aligned}
\zeta_{i}(t)= & -\sum_{j=1}^{m}\left[b_{j i}\left(\tilde{y}_{i}(t)\right)-b_{j i}\left(y_{i}(t)\right)\right] f_{j}\left(z_{j}(t)\right)-\sum_{j=1}^{m}\left[c_{j i}\left(\tilde{y}_{i}(t)\right)-c_{j i}\left(y_{i}(t)\right)\right] f_{j}\left(z_{j}(t-\rho(t))\right) \\
& -\sum_{j=1}^{m}\left[d_{j i}\left(\tilde{y}_{i}(t)\right)-d_{j i}\left(y_{i}(t)\right)\right] \int_{t-r(t)}^{t} f_{j}\left(z_{j}(s)\right) d s-\eta_{i}(t) \alpha_{i}(t) \\
\varsigma_{j}(t)= & -\sum_{i=1}^{n}\left[v_{i j}\left(\tilde{z}_{j}(t)\right)-v_{i j}\left(z_{j}(t)\right)\right] g_{i}\left(y_{i}(t)\right)-\sum_{i=1}^{n}\left[w_{i j}\left(\tilde{z}_{j}(t)\right)-w_{i j}\left(z_{j}(t)\right)\right] g_{i}\left(y_{i}(t-\rho(t))\right) \\
& -\sum_{i=1}^{n}\left[x_{i j}\left(\tilde{z}_{j}(t)\right)-x_{i j}\left(z_{j}(t)\right)\right] \int_{t-r(t)}^{t} g_{i}\left(y_{i}(s)\right) d s-\mu_{j}(t) \beta_{j}(t) .
\end{aligned}
$$

Let $\alpha_{i}(t)=\tilde{y}_{i}(t)+y_{i}(t)$ and $\beta_{j}(t)=\tilde{z}_{j}(t)+z_{j}(t)$. If $\tau=0$ and $\lambda=-1$, the control input (7) can be reduced into the following form:

$$
\begin{aligned}
\zeta_{i}(t)= & -\sum_{j=1}^{m} b_{j i}\left(y_{i}(t)\right) f_{j}\left(z_{j}(t)\right)-\sum_{j=1}^{m} c_{j i}\left(y_{i}(t)\right) f_{j}\left(z_{j}(t-\rho(t))\right)-\sum_{j=1}^{m} d_{j i}\left(y_{i}(t)\right) \int_{t-r(t)}^{t} f_{j}\left(z_{j}(s)\right) d s \\
& -\sum_{j=1}^{m} b_{j i}\left(\tilde{y}_{i}(t)\right) f_{j}\left(-z_{j}(t)\right)-\sum_{j=1}^{m} c_{j i}\left(\tilde{y}_{i}(t)\right) f_{j}\left(-z_{j}(t-\rho(t))\right) \\
& -\sum_{j=1}^{m} d_{j i}\left(\tilde{y}_{i}(t)\right) \int_{t-r(t)}^{t} f_{j}\left(-z_{j}(s)\right) d s-2 J_{i}-\eta_{i}(t) \alpha_{i}(t), \\
\varsigma_{j}(t)= & -\sum_{i=1}^{n} v_{i j}\left(z_{j}(t)\right) g_{i}\left(y_{i}(t)\right)-\sum_{i=1}^{n} w_{i j}\left(z_{j}(t)\right) g_{i}\left(y_{i}(t-\rho(t))\right)-\sum_{i=1}^{n} x_{i j}\left(z_{j}(t)\right) \int_{t-r(t)}^{t} g_{i}\left(y_{i}(s)\right) d s \\
& -\sum_{i=1}^{n} v_{i j}\left(\tilde{z}_{j}(t)\right) g_{i}\left(-y_{i}(t)\right)-\sum_{i=1}^{n} w_{i j}\left(\tilde{z}_{j}(t)\right) g_{i}\left(-y_{i}(t-\rho(t))\right) \\
& -\sum_{i=1}^{n} x_{i j}\left(\tilde{z}_{j}(t)\right) \int_{t-r(t)}^{t} g_{i}\left(-y_{i}(s)\right) d s-2 H_{j}-\mu_{j}(t) \beta_{j}(t) .
\end{aligned}
$$

Corollary 3. Supposing that Assumptions $[\mathcal{H}]$ hold and conditions of Theorem 3 hold, then systems (1) and (4) are globally projective synchronized, globally complete synchronized, globally anti-synchronized based on the hybrid controller (14) and (15), (16), (17) and (18), (19) when $\dot{\eta}_{i}(t), \dot{\mu}_{j}(t)$ designs as follows:

$$
\dot{\eta}_{i}(t)=\frac{-\kappa_{i} \theta_{i}\left|\alpha_{i}(t)\right|}{\eta_{i}(t)}+\kappa_{i}\left|\alpha_{i}(t)\right|, \dot{\mu}_{j}(t)=\frac{-\iota_{j} \delta_{j}\left|\beta_{j}(t)\right|}{\mu_{j}(t)}+\iota_{j}\left|\beta_{j}(t)\right|,
$$

where $\kappa_{i}, \theta_{i}, \xi_{i}, \iota_{j}, \delta_{j}$ and $\gamma_{j}$ denote the positive constants, $\eta_{i}(t)$ and $\mu_{j}(t)$ denotes the adaptive constants.

Proof. The proof of Corollary 3 is similar to the proof of Theorem 3. Hence, the proof is omitted here. 
Remark 3. In [54], the author considers lag-synchronization error systems as $\tilde{g}(e(t-\rho(t)))=g(y(t-$ $\rho(t)))-g(x(t-\sigma-\rho(t)))$, where $\sigma$ is lag transmittal delay. However, the author of [46] argues that the above synchronization error systems are not valid - thus corrected lag synchronization error systems such as $\tilde{g}(e(t-\rho(t)))=g(y(t-\rho(t)))-g(x(t-\sigma-\rho(t-\sigma)))$. In addition, the above consideration affects the validity of obtained proposed results. Here, evidently, we can choose the corrected lag synchronization errors with a projective sense. Thus, our error models are more valid and reasonable.

Remark 4. Suppose memristive connection weights satisfy these conditions $b_{j i}^{\star}=b_{j i}^{\star \star}, c_{j i}^{\star}=c_{j i}^{\star \star}, d_{j i}^{\star}=$ $d_{j i}^{\star \star}, v_{i j}^{\star}=v_{i j}^{\star \star}, w_{i j}^{\star}=w_{i j}^{\star \star}$ and $z_{i j}^{\star}=z_{i j}^{\star \star}$. Our problems turn up to the hybrid control for a general class of global projective lag synchronization of Riemann-Liouville type FOMBNNs with mixed time delays. In fact, we can easily derive from Theorem 3 to lag projective synchronization criteria for considered network models by employing the hybrid control method. When $q=1$, our result is still true for an integer order case.

Remark 5. In order to portray how to tune the control gains to achieve the projective lag synchronization goal for considered FOMBNNs, we take Theorem 3 and present some rules, which are listed below:

1. Based on the hybrid control, we choose the values of $\tau, \rho, \sigma, a_{i}, u_{j}, v_{i j}^{\star}, w_{j}^{\star}, x_{i j}^{\star}, b_{j i}^{\star}, c_{j i}^{\star}, d_{j i}^{\star}, L_{i}$ and $L_{j}$ according to assumptions and system parameters.

2. Next, justify whether

$$
\begin{aligned}
\theta_{i} & >\left[\sum_{j=1}^{m}\left(\mathbb{V}_{i j}+\frac{\mathbb{W}_{i j}}{1-\sigma}+r \mathbb{X}_{i j}\right) L_{i}-a_{i}\right], \\
\delta_{j} & >\left[\sum_{i=1}^{n}\left(\mathbb{B}_{j i}+\frac{\mathbb{C}_{j i}}{1-\sigma}+\rho \mathbb{D}_{j i}\right) \tilde{L}_{j}-u_{j}\right] .
\end{aligned}
$$

When we adjust the above parameters of the system, then the control gains of the hybrid controller (7) can be tuned slightly to realize a projective synchronization goal.

3. Next, we choose a time lag $\vartheta=2$ and projective coefficient $\lambda=3$.

4. Then, by using the dedicated simulation software tools and also selecting the simulation step size $h=0.01$, the output trajectories confirm that the tuned control gains converge gradually to some positive constants.

Remark 6. Basically, the Lyapunov method provides a very effective tool to realize synchronization analysis of a nonlinear system. However, it is very complicated to calculate the fractional order derivative of an auxiliary function. In order to avoid this complication, we design the integer order auxiliary function including fractional order derivative and integral terms and it helps to calculate its first-order derivative of an auxiliary function, which is guaranteed by the Definition of $R$-L fractional order differentiation and integration. The main advantage of our proposed method is that we can avoid calculating the fractional-order derivatives of the Lyapunov functional.

\section{Numerical Example}

Consider a vector form of two-state FOMBNNs as follows:

$$
\begin{aligned}
& D^{q} y(t)=-A y(t)+B(y(t)) f(z(t))+C(y(t)) f(z(t-\rho(t)))+D(y(t)) \int_{t-r(t)}^{t} f(z(s)) d s+J, \\
& D^{q} z(t)=-U z(t)+V(z(t)) g(y(t))+W(z(t)) g(y(t-\rho(t)))+X(z(t)) \int_{t-r(t)}^{t} g(y(s)) d s+H,
\end{aligned}
$$


where $q=0.97, y(t)=\left(y_{1}(t), y_{2}(t)\right)^{T}, z(t)=\left(z_{1}(t), z_{2}(t)\right)^{T}, f(z)=\left(f_{1}\left(z_{1}\right), f_{2}\left(z_{2}\right)\right)^{T}, g(y)=$ $\left(g_{1}\left(y_{1}\right), g_{2}\left(y_{2}\right)\right)^{T}, f_{j}\left(z_{j}\right)=\tanh \left(z_{j}\right), g_{i}\left(y_{i}\right)=\tanh \left(y_{i}\right), \rho(t)=\frac{0.8 e^{t}}{1+e^{t}}, r(t)=0.5 \cos t+1, \sigma=0.2<$ $1, r=1, \rho=2, J=\left(\begin{array}{ll}0 & 0\end{array}\right)^{T}, H=\left(\begin{array}{ll}0 & 0\end{array}\right)^{T}, a_{1}=a_{2}=4, u_{1}=u_{2}=5$, and

$$
\begin{aligned}
& B(y(t))=\left(\begin{array}{cc}
b_{11}\left(y_{1}\right) & -3 \\
2 & b_{22}\left(y_{2}\right)
\end{array}\right), C(y(t))=\left(\begin{array}{cc}
c_{11}\left(y_{1}\right) & 1 \\
-5 & c_{22}\left(y_{2}\right)
\end{array}\right), D(y(t))=\left(\begin{array}{cc}
d_{11}\left(y_{1}\right) & 3 \\
-2 & d_{22}\left(y_{2}\right)
\end{array}\right), \\
& V(z(t))=\left(\begin{array}{cc}
v_{11}\left(z_{1}\right) & -1.8 \\
-2.5 & v_{22}\left(z_{2}\right)
\end{array}\right), W(z(t))=\left(\begin{array}{cc}
w_{11}\left(z_{1}\right) & -2.8 \\
4.5 & w_{22}\left(z_{2}\right)
\end{array}\right), X(z(t))=\left(\begin{array}{cc}
x_{11}\left(z_{1}\right) & -3.8 \\
2 & x_{22}\left(z_{2}\right)
\end{array}\right) \text {, } \\
& b_{11}\left(y_{1}(t)\right)=\left\{\begin{array}{cc}
2.31, & \left|y_{1}(t)\right| \leq 1 \\
-2.31, & \left|y_{1}(t)\right|>1,
\end{array} \quad b_{22}\left(y_{2}(t)\right)=\left\{\begin{array}{cc}
2, & \left|y_{2}(t)\right| \leq 1 \\
-2, & \left|y_{2}(t)\right|>1
\end{array}\right.\right. \\
& c_{11}\left(y_{1}(t)\right)=\left\{\begin{array}{cc}
1.62, & \left|y_{1}(t)\right| \leq 1 \\
-1.62, & \left|y_{1}(t)\right|>1,
\end{array} \quad c_{22}\left(y_{2}(t)\right)=\left\{\begin{array}{cc}
2.5, & \left|y_{2}(t)\right| \leq 1 \\
-2.5, & \left|y_{2}(t)\right|>1,
\end{array}\right.\right. \\
& d_{11}\left(y_{1}(t)\right)=\left\{\begin{array}{cc}
1.3, & \left|y_{1}(t)\right| \leq 1 \\
-1.3, & \left|y_{1}(t)\right|>1,
\end{array} \quad d_{22}\left(y_{2}(t)\right)=\left\{\begin{array}{cc}
1.72, & \left|y_{2}(t)\right| \leq 1 \\
-1.72, & \left|y_{2}(t)\right|>1
\end{array}\right.\right. \\
& v_{11}\left(z_{1}(t)\right)=\left\{\begin{array}{cl}
0.81, & \left|z_{1}(t)\right| \leq 1 \\
-0.81, & \left|z_{1}(t)\right|>1,
\end{array} \quad v_{22}\left(z_{2}(t)\right)=\left\{\begin{array}{cc}
0.88, & \left|z_{2}(t)\right| \leq 1 \\
-0.88, & \left|z_{2}(t)\right|>1
\end{array}\right.\right. \\
& w_{11}\left(z_{1}(t)\right)=\left\{\begin{array}{cl}
1.37, & \left|z_{1}(t)\right| \leq 1 \\
-1.37, & \left|z_{1}(t)\right|>1,
\end{array} \quad w_{22}\left(z_{2}(t)\right)=\left\{\begin{array}{cc}
1.03, & \left|z_{2}(t)\right| \leq 1 \\
-1.03, & \left|z_{2}(t)\right|>1
\end{array}\right.\right. \\
& x_{11}\left(z_{1}(t)\right)=\left\{\begin{array}{cc}
1.21, & \left|z_{1}(t)\right| \leq 1 \\
-1.21, & \left|z_{1}(t)\right|>1,
\end{array} \quad x_{22}\left(z_{2}(t)\right)=\left\{\begin{array}{cc}
0.42, & \left|z_{2}(t)\right| \leq 1 \\
-0.42, & \left|z_{2}(t)\right|>1 .
\end{array}\right.\right.
\end{aligned}
$$

The response system is given by

$$
\begin{aligned}
& D^{q} \tilde{y}(t)=-A \tilde{y}(t)+B(\tilde{y}(t)) f(\tilde{z}(t))+C(\tilde{y}(t)) f(\tilde{z}(t-\rho(t)))+D(\tilde{y}(t)) \int_{t-r(t)}^{t} f(\tilde{z}(s)) d s+J+\zeta(t) \\
& D^{q} \tilde{z}(t)=-U \tilde{z}(t)+V(\tilde{z}(t)) g(\tilde{y}(t))+W(\tilde{z}(t)) g(\tilde{y}(t-\rho(t)))+X(\tilde{z}(t)) \int_{t-r(t)}^{t} g(\tilde{y}(s)) d s+H+\varsigma(t),(21)
\end{aligned}
$$

where $\zeta(t)=\left(\zeta_{1}(t), \zeta_{2}(t)\right)^{T}$ and $\varsigma(t)=\left(\varsigma_{1}(t), \varsigma_{2}(t)\right)^{T}$. All other parameters are similar to that defined in a drive system. In hybrid control scheme $(7)$, we can choose $\eta_{i}(0)=\mu_{j}(0)=0.01, \kappa_{i}=\theta_{i}=0.1, \iota_{j}=\delta_{i}=$ 0.1 for $i, j=1,2 . \theta_{1}=11.6, \theta_{2}=11.5, \delta_{1}=7, \delta_{2}=7.5$ and $\lambda=3, L_{1}=L_{2}=0.8, \tilde{L}_{1}=0.5, \tilde{L}_{2}=0.4$. It is easy to check the following inequities hold 


$$
\begin{aligned}
& \min _{i}\left\{a_{i}+\theta_{i}-\sum_{j=1}^{m}\left(\mathbb{V}_{i j}+\frac{\mathbb{W}_{i j}}{1-\sigma}+r \mathbb{X}_{i j}\right) L_{i}\right\}>0, \\
& \min _{j}\left\{u_{j}+\delta_{j}-\sum_{i=1}^{n}\left(\mathbb{B}_{j i}+\frac{\mathbb{C}_{j i}}{1-\sigma}+r \mathbb{D}_{j i}\right) \tilde{L}_{j}\right\}>0
\end{aligned}
$$

for $i, j=1$, 2. Based on Theorem 3, the drive-response systems (20) and (21) are globally projective lag synchronized via hybrid controller (7). Next, we select the initial values of FOMBNNs (20) and (21) as $(y(t), z(t))=(1.8,-1.5,-1.9,-0.5)$ and $(\tilde{y}(t), \tilde{z}(t))=(-1.2,1.1,-1.1,-1.2)$. The projective lag synchronization error evolution for state variables are depicted in Figures 1 and 2, which shows that synchronization of projective error dynamical systems converges to origin, while the adaptive control gains are provided in Figures 3 and 4, which confirms that the adaptive control gains converge to some positive constants.

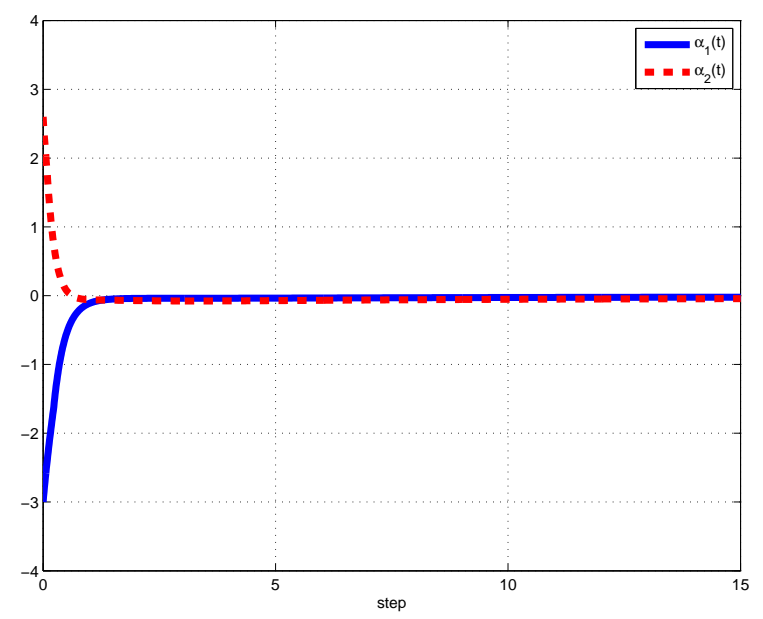

Figure 1. The error state trajectory of $r_{i}(t)$ and $\tilde{r}_{j}(t)$.

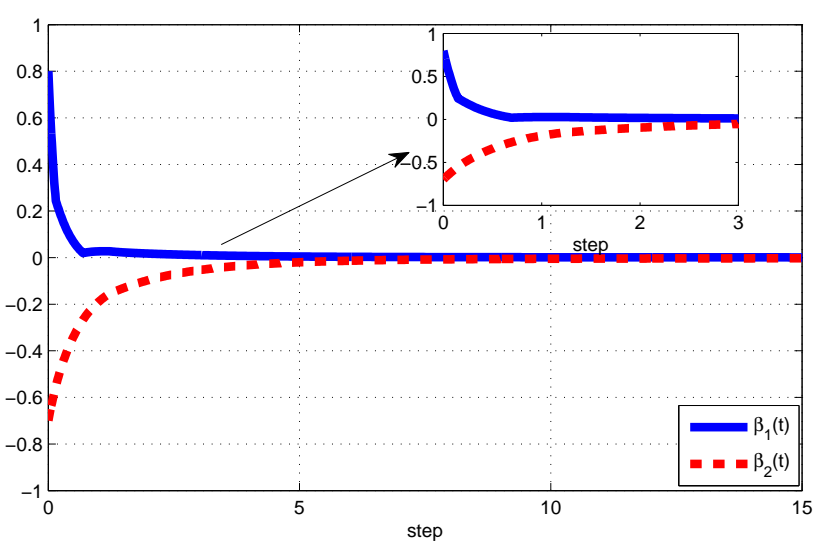

Figure 2. The error state trajectory of $r_{i}(t)$ and $\tilde{r}_{j}(t)$. 


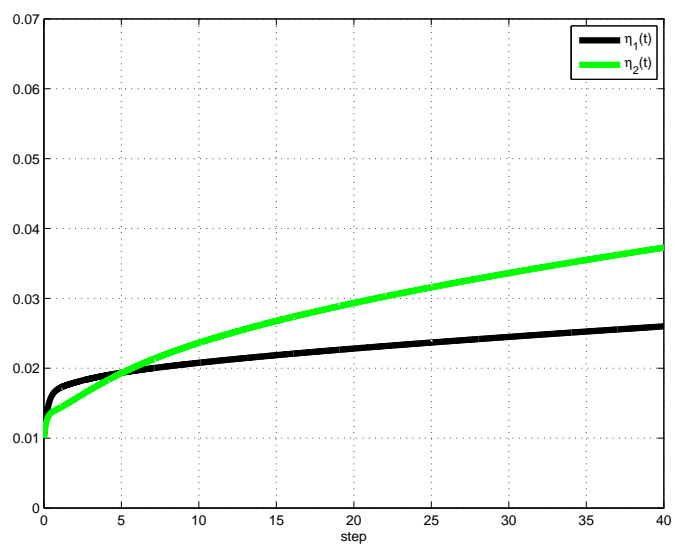

Figure 3. The control gains of controller (7).

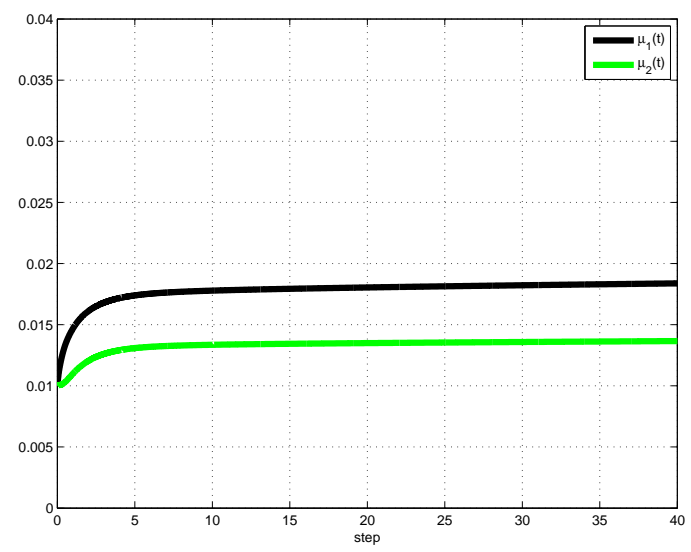

Figure 4. The control gains of controller (7).

\section{Conclusions}

In this research work, a hybrid control scheme for projective lag synchronization of FOMBNNs with mixed delays is investigated. On the one hand, by a key role of Lyapunov theory, differential inclusion theory and fractional order Barbalet's lemma, some novel sufficient conditions have been established to assure the projective lag synchronization of the designed fractional order neural network model. On the other hand, as a special case of complete synchronization, anti-synchronization and projective synchronization of FOMBNNs are also investigated, and these results have not been seen yet. Lastly, we give some computer simulations to delineate the correctness of the proposed main consequences.

In $[50,54,55]$, the authors discussed lag synchronization of integer and fractional order memristive neural networks with switching jump mismatch, based on different control approaches including a state feedback control, period intermittent control. In [56,57], authors proposed the projective synchronization with (or without) memristive neural networks via hybrid control schemes in the Caputo sense. Inspired by [21], the author derived asymptotic stability conditions of delayed FOMBNNs via fractional Barbalat's Lemma and R-L derivative properties. However, the hybrid control for projective lag synchronization with the combination of the Riemann-Liouville sense fractional order memristive BAM neural networks model is discussed for the first time in this work. Moreover, the presented hybrid control can be applied for solving projective lag synchronization of Riemann-Liouville sense fractional order Cohen-Grossberg coupled neural networks with mixed delays, and we will consider this interesting issue for future work. 
Author Contributions: All the authors have contributed equally in the article.

Funding: This work was partially supported by the National Natural Science Foundation of P. R. China (Nos. 11971076, 71861008).

Acknowledgments: This article has been written with the joint financial support of RUSA-Phase 2.0 Grant No.F 24-51/2014-U, Policy (TN Multi-Gen), Dept. of Edn. Govt. of India, UGC-SAP (DRS-I) Grant No.F.510/8/DRS-I/ 2016(SAP-I) and DST (FIST-level I) 657876570 Grant No.SR/FIST/MS-I/2018/17. Moreover, J. Alzabut would like to thank Prince Sultan University for funding this work through research group Nonlinear Analysis Methods in Applied Mathematics (NAMAM) group number RG-DES-2017-01-17.

Conflicts of Interest: The authors declare no conflict of interest.

\section{References}

1. Huang, C.; Liu, B. New studies on dynamic analysis of inertial neural networks involving non-reduced order method. Neurocomputing 2019, 325, 283-287. [CrossRef]

2. Wang, J.; Huang, C.; Huang, L. Discontinuity-induced limit cycles in a general planar piecewise linear system of saddle-focus type. Nonlinear Anal. Hybrid Syst. 2019, 33, 162-178. [CrossRef]

3. Wang, J.; Chen, X.; Huang, L. The number and stability of limit cycles for planar piecewise linear systems of node-saddle type. J. Math. Anal. Appl. 2019, 469, 405-427. [CrossRef]

4. Chen, T.; Huang, L.; Yu, P.; Huang, W. Bifurcation of limit cycles at infinity in piecewise polynomial systems. Nonlinear Anal. Real World Appl. 2018, 41, 82-106. [CrossRef]

5. Cai, Z.; Huang, J.; Huang, L. Periodic orbit analysis for the delayed Filippov system. Proc. Am. Math. Soc. 2018, 146, 4667-4682. [CrossRef]

6. Yang, C.; Huang, L.; Li, F. Exponential synchronization control of discontinuous nonautonomous networks and autonomous coupled networks. Complexity 2018, 2018, 6164786. [CrossRef]

7. Zuo, Y.; Wang, Y.; Liu, X. Adaptive robust control strategy for rhombus-type lunar exploration wheeled mobile robot using wavelet transform and probabilistic neural network. Comput. Appl. Math. 2018, 37, 314-337. [CrossRef]

8. $\mathrm{Hu}, \mathrm{H} . ; \mathrm{Zou}, \mathrm{X}$. Existence of an extinction wave in the fisher equation with a shifting habitat. Proc. Am. Math. Soc. 2017, 145, 4763-4771. [CrossRef]

9. Song, C.; Fei, S.; Cao, J.; Huang, C. Robust Synchronization of Fractional-Order Uncertain Chaotic Systems Based on Output Feedback Sliding Mode Control. Mathematics 2019, 7, 599. [CrossRef]

10. Cai, Z.; Huang, J.; Huang, L. Generalized Lyapunov-Razumikhin method for retarded differential inclusions: Applications to discontinuous neural networks. Discret. Contin. Dyn. Syst. Ser. B 2017, 22, 3591-3614. [CrossRef]

11. Tan, Y.; Zhang, M. Global exponential stability of periodic solutions in a nonsmooth model of hematopoiesis with time-varying delays. Math. Methods Appl. Sci. 2017, 40, 5986-5995. [CrossRef]

12. Huang, C.; Cao, J.; Wen, F.; Yang, X. Stability Analysis of SIR Model with Distributed Delay on Complex Networks. PLoS ONE 2016, 11, e0158813. [CrossRef] [PubMed]

13. Diethelm, K. The Analysis of Fractional Differential Equations; Springer: Berlin, Germany, 2010.

14. Hilfer, R. Applications of Fractional Calculus in Physics; World Scientific: Singapore, 2000; Volume 128.

15. Kilbas, A.; Srivastava, A.; Trujillo, J.J. Theory and Applications of Fractional Differential Equations; Elsevier Science Limited: Amsterdam, The Netherlands, 2006; Volume 204.

16. Podlubny, I. Fractional Differential Equations; Academic Press: San Diego, CA, USA, 1999.

17. Laskin, N. Fractional market dynamics. Phys. A Stat. Mech. Appl. 2000, 287, 482-492. [CrossRef]

18. Lundstrom, B.N.; Higgs, M.H.; Spain, W.J.; Fairhall, A.L. Fractional differentiation by neocortical pyramidal neurons. Nat. Neurosci. 2008, 11, 1335-1342. [CrossRef] [PubMed]

19. Petras, I. Fractional-Order Nonlinear Systems: Modeling, Analysis and Simulation; Springer: Berlin, Germany, 2011. 
20. Ali, S.; Narayanan, G.; Sevgen, S.; Sheher, V.; Arik, S. Global stability analysis of fractional-order fuzzy BAM neural networks with time delay and impulsive effects. Commun. Nonlinear Sci. Numer. Simul. 2019, 78,104853

21. Wang, F.; Yang, Y.; Xu, X.; Li, L. Global asymptotic stability of impulsive fractional-order BAM neural networks with time delay. Neural Comput. Appl. 2017, 28, 345-352. [CrossRef]

22. Wu, A.; Zeng, Z.; Song Global, S. Mittag-Leffler stabilization of fractionalorder bidirectional associative memory neural networks. Neurocomputing 2016, 177, 489-496. [CrossRef]

23. Ye, R.; Liu, X.; Zhang, H.; Cao, J. Global Mittag-Leffler synchronization for fractional-order BAM neural networks with impulses and multiple variable delays via delayed-feedback control strategy. Neural Process. Lett. 2019, 49,1-18. [CrossRef]

24. Rajivganthi, C.; Rihan, F.; Laxshmanan, S.; Rakkiappan, R.; Muthuumar, P. Synchronization of memristor-based delayed BAM neural networks with fractional-order derivatives. Complexity 2016, 21, 412-426. [CrossRef]

25. Duan, L.; Fang, X.; Huang, C. Global exponential convergence in a delayed almost periodic Nicholson's blowflies model with discontinuous harvesting. Math. Methods Appl. Sci. 2018, 41, 1954-1965. [CrossRef]

26. Huang, C.; Liu, B.; Tian, X.; Yang, L.; Zhang, X. Global convergence on asymptotically almost periodic SICNNs with nonlinear decay functions. Neural Process. Lett. 2019, 49, 625-641. [CrossRef]

27. Huang, C.; Zhang, H.; Huang, L. Almost periodicity analysis for a delayed Nicholson's blowflies model with nonlinear density-dependent mortality term. Commun. Pure Appl. Anal. 2019, 18, 3337-3349. [CrossRef]

28. Yang, X.; Zhu, Q.; Huang, C. Generalized lag-synchronization of chaotic mix-delayed systems with uncertain parameters and unknown perturbations. Nonlinear Anal. Real World Appl. 2011, 12, 93-105. [CrossRef]

29. Huang, C.; Qiao, Y.; Huang, L. Agarwal, Dynamical behaviors of a food-chain model with stage structure and time delays. Adv. Differ. Equ. 2018, 2018, 186. [CrossRef]

30. Huang, C.; Zhang, H. Periodicity of non-autonomous inertial neural networks involving proportional delays and non-reduced order method. Int. J. Biomath. 2019, 12, 1950016. [CrossRef]

31. Zhu, Q.; Huang, C.; Yang, X. Exponential stability for stochastic jumping BAM neural networks with time-varying and distributed delays. Nonlinear Anal. Hybrid Syst. 2011, 5, 52-77. [CrossRef]

32. Huang, C.; Su, R.; Cao, J.; Xiao, S. Asymptotically stable high-order neutral cellular neural networks with proportional delays and D operators. Math. Comput. Simul. 2019. [CrossRef]

33. Huang, C.; Zhang, H.; Cao, J.; Hu, H. Stability and Hopf bifurcation of a delayed prey-predator model with disease in the predator. Int. J. Bifurc. Chaos 2019, 29, 1950091. [CrossRef]

34. Huang, C.; Yang, Z.; Yi, T.; Zou, X. On the basins of attraction for a class of delay differential equations with non-monotone bistable nonlinearities. J. Differ. Equ. 2014, 256, 2101-2114. [CrossRef]

35. Wu, H.; Zhang, X.; Xue, S.; Niu, P. Quasi-uniform stability of Caputo-type fractional-order neural networks with mixed delay. Int. J. Mach. Learn. Cybern. 2017, 8, 1501-1511. [CrossRef]

36. Zhang, H.; Ye, R.; Cao, J.; Alsaedi, A. Existence and globally asymptotic stability of equilibrium solution for fractional-order hybrid BAM neural networks with distributed delays and impulses. Complexity 2017, 2017, 6875874. [CrossRef]

37. Chua, L.Q. Memristor-the missing circuit element. IEEE Trans. Circuit Theory 1971, 18, 507-519. [CrossRef]

38. Strukov, D.; Snider, G.; Stewart, D.; Williams, R. The missing memristor found. Nature 2008, 453, 80-83. [CrossRef]

39. Kim, H.; Sah, M.P.; Yang, C.; Roska, T.; Chua, L.O. Memristor bridge synapses. Proc. IEEE 2012, 100, 2061-2070. [CrossRef]

40. Bao, H.B.; Cao, J.; Kurths, J. State estimation of fractional-order delayed memristive neural networks. Nonlinear Dyn. 2018, 2, 1215-1225. [CrossRef]

41. Chang, W.; Zhu, S.; Li, J.; Sun, K. Global Mittag-Leffler stabilization of fractional-order complex-valued memristive neural networks. Appl. Math. Comput. 2018, 338, 346-362. [CrossRef] 
42. Wu, A.; Zeng, Z. Global Mittag-Leffler stabilization of fractional-Order memristive neural networks. IEEE Trans. Neural Netw. Learn. Syst. 2017, 28, 206-217. [CrossRef]

43. Li, X.; Fang, J.A.; Zhang, W.; Li, H. Finite-time synchronization of fractional-order memristive recurrent neural networks with discontinuous activation functions. Neurocomputing 2018, 316, 284-293. [CrossRef]

44. Pecora, L.; Carrol, T. Synchronization in chaotic systems. Phys. Rev. Lett. 1990, 64, 821-824. [CrossRef]

45. Liu, X.; Jiang, N.; Cao, J. Finite-time stochastic stabilization for BAM neural networks with uncertainties. J. Frankl. Inst. 2013, 350, 2109-2123. [CrossRef]

46. Abdurahman, A.; Jiang, H.; Teng, Z. Exponential lag synchronization for memristor-based neural networks with mixed time delays via hybrid switching control. J. Frankl. Inst. 2016, 353, 2859-2880. [CrossRef]

47. Velmurugan, G.; Rakkiyappan, R. Hybrid projective synchronization of fractional-order memristor-based neural networks with time delays. Nonlinear Dyn. 2016, 83, 419-432. [CrossRef]

48. Wu, H.; Wang, L.; Niu, P.; Wang, Y. Global projective synchronization in finite time of nonidentical fractional order neural networks based on sliding mode control strategy. Neurocomputing 2017, 235, 264-273. [CrossRef]

49. Xiao, J.; Zhong, S.; Li, Y.; Xu, F. Finite-time Mittag-Leffler synchronization of fractional-order memristive BAM neural networks with time delays. Neurocomputing 2016, 219, 431-439. [CrossRef]

50. Zhang, L.; Yang, Y.; Wang, F. Lag synchronization for fractional-order memristive neural networks via period intermittent control. Nonlinear Dyn. 2017, 89, 367-381. [CrossRef]

51. Popov, V. Hyperstability of Control Systems; Springer: Berlin, Germany, 1973.

52. Filippov, A.F. Differential Equations with Discontinuous Right-Hand Sides; Kluwer: Dordrecht, The Netherlands, 1988.

53. Henderson, J.; Ouahab, A. Fractional functional differential inclusions with finite delay. Nonlinear Anal. Theory Methods Appl. 2009, 70, 2091-2105. [CrossRef]

54. Li, N.; Cao, J.D. Lag synchronization of memristor-based coupled neural networks via $\omega$-measure. IEEE Trans. Neural Netw. Learn. Syst. 2016, 27, 686-697. [CrossRef]

55. Ding, S.B.; Wang, Z.S. Lag quasi-synchronization for memristive neural networks with switching jumps mismatch. Neural Comput. Appl. 2017, 28, 4011-4022. [CrossRef]

56. Bao, H.B.; Cao, J. Projective synchronization of fractional-order memristor-based neural networks. Neural Netw. 2015, 63, 1-9. [CrossRef]

57. Yu, J.; Hu, C.; Jiang, H.; Fan, X. Projective synchronization for fractional neural networks. Neural Netw. 2014, 49, 87-95. [CrossRef]

(C) 2019 by the authors. Licensee MDPI, Basel, Switzerland. This article is an open access article distributed under the terms and conditions of the Creative Commons Attribution (CC BY) license (http:/ / creativecommons.org/licenses/by/4.0/). 